Sharif University of Technology
Scientia Iranica
SCIENTIA

\title{
Chaotic behaviors of a ground vehicle oscillating system with passengers
}

\author{
J. Fakhraei ${ }^{a}$, H.M. Khanlo ${ }^{\text {b,* }}$ and M. Ghayour ${ }^{a}$ \\ a. Department of Mechanical Engineering, Isfahan University of Technology, Isfahan, P.O. Box 84156-83111, Iran. \\ b. Department of Aerospace Engineering, Shahid Sattari Aeronautical University of Science and Technology, Tehran, P.O. Box \\ 13846-63113, Iran
}

Received 29 May 2015; received in revised form 25 June 2016; accepted 3 September 2016

\section{KEYWORDS}

Dynamic behavior;

Chaotic;

Time delay;

Vehicle;

Bifurcation.

\begin{abstract}
In this paper, some considerations regarding a ground vehicle oscillating system based on chaotic behaviors are studied. The vehicle system is modeled as a full nonlinear seven-degree freedom with an additional degree of freedom for each passenger. Roughness of the road surface is considered as sinusoidal waveforms with time delays for the tires. The governing differential equations are extracted under Newton-Euler laws and solved via numerical methods. The dynamic behavior of the system is investigated by special nonlinear techniques such as bifurcation diagram, time series, phase plane portrait, power spectrum, Poincaré section, and maximum Lyapunov exponents. The time delays between the tires are used as a control parameter. First, the vehicle behavior is investigated and the chaotic regions are detected. Then, the damping and stiffness coefficients are used to return to the regular behavior. Results show that by changing the system parameters and selecting the appropriate values, one can minimize vibrations as well as eliminate chaotic behavior. The comparison of the results obtained from the proposed model and those from the vehicle without passengers show the great differences in the dynamic behaviors of the two models.

(C) 2017 Sharif University of Technology. All rights reserved.
\end{abstract}

\section{Introduction}

Unwanted vehicle vibrations caused by road surface roughness are still the subject of many research studies among automotive manufacturers and research groups whose objective is to minimize their effects on the passengers and increase the life of vehicle components. Also, the controllability and stability of ground vehicles facing with road surface roughness is an essential consideration in design of vehicle dynamics. In order to analyzes vehicle dynamics, the vehicle is considered as

*. Corresponding author. Tel.: +982164032302;

Fax: +982164032302

E-mail addresses: j_fakhraee@yahoo.com (J. Fakhraei);

H_khanloo@ssau.ac.ir (H.M.Khanlo); ghayour@cc.iut.ac.ir (M. Ghayour) an oscillating system which consists of three essential components: the main body of vehicle (sprung mass), the wheels (unsprung mass), and the suspension system. On the other hand, the presence of the passenger as an unavoidable factor in a moving vehicle plays an important role in the dynamic responses. Hence, it seems that consideration of a suitable model for realizing the interaction between passenger and vehicle is necessary. However, most previous studies have investigated the dynamic behavior of vehicle without any consideration for passengers. So, in this paper, the dynamic behavior and vibration response of a vehicle with passengers are analyzed. The dynamic behavior of vehicle systems has been the subject of many studies over the past decade. In this regard, three typical models have been developed. They are the quarter-car, half-car, full vehicle models as single-wheel, two-wheel, 
and four-wheel models, respectively. The quarter-car model (1- or 2-d.o.f. system) is used only when heave motion needs to be considered [1-4]. The half-car model (4-d.o.f. system) as a two-wheel (front and rear) one is used to study heave and pitch motions with the deflection of tires and suspension [5-7]. A more complex model is the full vehicle one that is a $3-\mathrm{D}$ model with seven degrees of freedom that can be used for studying heave, pitch, and roll motions [8]. Therefore, this model has a more accurate dynamic response than those of the other two.

In the study of vehicle dynamics, there are some contradictions between experimental results and those obtained from linear models. The nonlinear characteristics of vehicle components are the source of these contradictions in linear models. Many nonlinear units are used to reduce the vibration and impact of modern vehicles, e.g. magneto-rheological damper and unequal curvature spring [9-11]. Owing to the existence of the nonlinear factor, the vehicle exhibits complex phenomena, such as jumps, bifurcation, and chaotic vibrations, when running on a bumpy road that is quite harmful for the stabilization of a vehicle. In recent years, many studies have developed these phenomena in a variety of vehicle models with various degrees of freedom. Litak et al. [12-14] investigated chaotic vibrations in a quarter-car model with one degree of freedom under the sinusoidal excitation force of road. The stability and chaos analyses of a nonlinear quarter-vehicle model with two degrees of freedom were investigated by Samandari and Rezaee [15]. Their results showed that the system response is sensitive to road roughness amplitude, the excitation frequency (vehicle velocity), and the initial conditions of the system. Singru and Naik [16] studied the resonance, stability, and chaotic vibrations of a quarter-car model with time delay feedback. Their results showed that appropriate feedback and time-delay can enhance control performance in the case of primary and super harmonic resonances. For sub-harmonic resonance, adequate feedback can eliminate the occurrence of subharmonic resonance response. Zhu and Ishitobi [17] studied the chaotic responses and bifurcations of a halfvehicle model with four degrees of freedom subjected to two sinusoid disturbances with a time delay. In their study, the bifurcation diagram shows that the chaotic response could be sensitive to a variation in the damping of the suspension. Also, in another study, the chaotic vibrations of a nonlinear full-vehicle model with seven degrees of freedom were studied [18]. By using frequency response diagrams, they showed that the number of unstable regions can be decreased by increasing the forcing frequency. This is a typical phenomenon for nonlinear dynamic systems. However, this phenomenon was not observed in the dynamic responses of the vehicle model with two or four degrees of freedom. Fakhraei et al. [19] studied the effect of passengers on the chaotic vibrations of a nonlinear full vehicle model. The results obtained represent different types of strange attractors in the vehicle with and without passengers. Also, taking the passengers into consideration and increasing the mass of the system can result in significant changes in the dynamic behavior of the system and improves the chaotic vibration of the vehicle. Sedighi and Shirazi [20] investigated the bifurcation of railway bogie behavior in the presence of nonlinearities which are yaw damping forces in longitudinal suspension system and the friction creepage model of the wheel/rail contact including clearance. By using two specific criteria, the analytical expression of critical speed and the limit cycle frequency are achieved. Also, by averaging method and analytical critical speed, the amplitude of the limit cycle is determined while the wheel/rail clearance is taken into account.

In this paper, a nonlinear full vehicle model with seven degrees of freedom is considered, and passengers are added to it. Then, the chaotic vibrations in the nonlinear full vehicle model with passengers are analyzed by using the identification techniques of chaotic motion. The effects of the main parameters on chaotic vibrations are investigated in the following sections.

\section{Mathematical model}

Figure 1 shows the schematic of a nonlinear full-vehicle model with passengers. This system has eleven degrees of freedom, and the definitions of used symbols are given in the appendix. Each passenger is in a seat modeled by a block with mass, $m_{p}$, connected to a linear spring and damper and is free to bounce vertically. The vehicle body is represented by rigid cuboids with three degrees of freedom and with mass $m_{s}$. The heave, pitch, and roll motions of the sprung mass are considered. The four unsprung masses (frontleft, front-right, rear-left, and rear-right) are connected to each corner of the rigid cuboids. It is assumed that the four unsprung masses are free to bounce vertically. The suspension between the sprung mass and the unsprung masses is modeled as nonlinear spring and nonlinear damper elements, while tires are modeled as nonlinear springs with viscous damping.

The forces of the linear spring attached to the seats are given by:

$$
F_{p k}=k_{p} \Delta_{p}, \quad p=a, b, c, d,
$$

where $F_{p k}$ is the spring dynamic force, $k_{p}$ is the equivalent stiffness, $\Delta_{p}$ is the deformation of the spring. Subscript $p$ indicates the position of the passenger on the vehicle body. The linear damping forces of the passenger seat are given as:

$$
F_{p c}=c_{p} \dot{\Delta}_{p}, \quad p=a, b, c, d,
$$




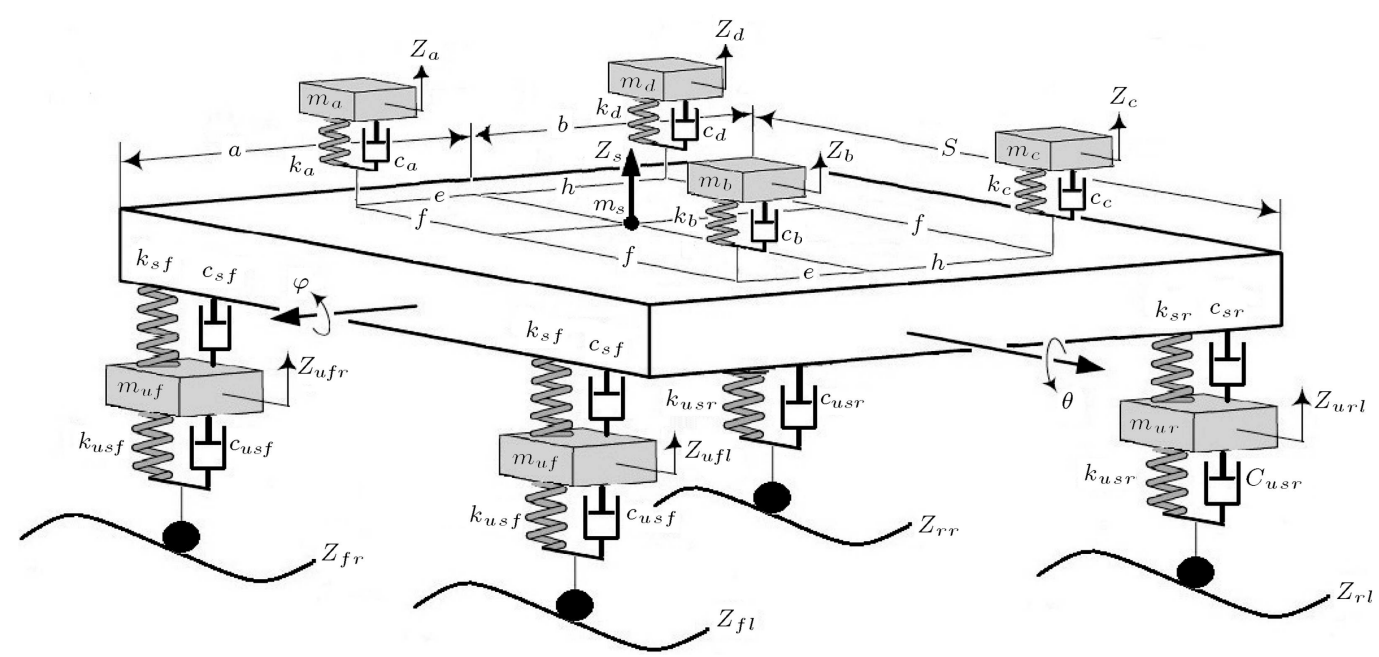

Figure 1. Schematic of nonlinear full-vehicle model with passengers.

where $F_{p c}$ is the damping force, $c_{p}$ is the equivalent damping coefficient, and $\dot{\Delta}_{p}$ is the relative velocity of the damper.

It is assumed that the modeled nonlinear suspension spring has the following characteristics [5]:

$$
\begin{aligned}
& F_{s i j}=k_{s i j} \operatorname{sgn}\left(\Delta_{s i j}\right)\left|\Delta_{s i j}\right|^{n_{s i j}} \\
& (i=f, r, \quad j=r, l),
\end{aligned}
$$

where $F_{s i j}$ is the spring dynamic force, $k_{s i j}$ is the equivalent stiffness, $\Delta_{s i j}$ is the deformation of the spring, and $\operatorname{sgn}($.$) is the sign function. Subscript s$ represents the spring of suspension, the subscript $i=f, r$ indicates the front and rear, while the subscript $j=l, r$, indicates the left and right. Also, $F_{s f r}, k_{s f r}$, and $\Delta_{s f r}$ indicate the spring force, equivalent stiffness, and deformation of the suspension spring in the front and right corners, respectively. In Eq. (3), $n_{s i j}$ is an exponent which represents the nonlinearity of the spring, and it is termed as the nonlinear coefficient. The unit of $\Delta_{s i j}$ is in $\mathrm{cm}$ and $k_{s i j}$ in $\mathrm{N} / \mathrm{cm}$. Since the suspensions are usually arranged symmetrically along the longitudinal axis of the vehicle, Eq. (3) can be written as:

$$
F_{s i j}=k_{s i} \operatorname{sgn}\left(\Delta_{s i j}\right)\left|\Delta_{s i j}\right|^{n_{s i}} \quad(i=f, r, \quad j=r, l) .
$$

The nonlinear damping forces of the front and rear suspensions are given as:

$$
F_{c i j}=c_{s i} \dot{\Delta}_{u i j} \quad(i=f, r, \quad j=r, l)
$$

where subscript $c$ indicates the damping of the suspension, $F_{c i j}$ is the damping force, and $\dot{\Delta}_{u i j}$ is the relative velocity between the extremes of the damper. Damping coefficient $c_{s i}$ is expressed as:

$$
c_{s i}=\left\{\begin{array}{ll}
c_{s u i} & \dot{\Delta}_{u i j} \geq 0 \\
c_{s d i} & \dot{\Delta}_{u i j}<0
\end{array} \quad(i=f, r, \quad j=r, l),\right.
$$

where $c_{\text {sui }}$ and $c_{\text {sdi }}$ are the damping coefficients for tension and compression, respectively.

The tire of vehicle is also modeled by a nonlinear spring, and the spring force is the same as Eq. (4) but with a smaller value for the nonlinear coefficient:

$$
\begin{gathered}
F_{u s i j}=k_{u s i} \operatorname{sgn}\left(\Delta_{u s i j}\right)\left|\Delta_{u s i j}\right|^{n_{u s i}} \\
(i=f, r, \quad j=r, l)
\end{gathered}
$$

where $F_{u s i j}$ is the spring force, $k_{u s i j}$ is the equivalent stiffness, $\Delta_{u s i j}$ is the deformation, and $n_{u s i j}$ is the nonlinear coefficient of the tire spring.

It is assumed that the damping of the tires is viscous; thus, the damping force is:

$$
F_{u c i j}=c_{u s i} \dot{\Delta}_{u s i j} \quad(i=f, r, \quad j=r, l),
$$

where $c_{u s i}$ is the viscous damping coefficient, and $\dot{\Delta}_{u s i j}$ is the relative velocity of the extremes of the tire model.

The sinusoid forcing function is used to describe the excitations caused by the road surface. Thus, the forcing functions for the tires in front-right, frontleft, rear-right, and rear-left unsprung masses are approximated as [18]:

$$
\begin{aligned}
& Z_{f r}=A \sin (2 \pi f t), \\
& Z_{f l}=A \sin (2 \pi f t+\beta), \\
& Z_{r r}=A \sin (2 \pi f t+\alpha), \\
& Z_{r l}=A \sin (2 \pi f t+\beta+\alpha),
\end{aligned}
$$

where $A$ and $f$ are the amplitude and the frequency of the sinusoid road disturbance, respectively. Parameter $\beta$ indicates the time delay between the forcing functions of two front tires, or to two rear tires, respectively. Also, $\alpha$ indicates the time delay between the forcing functions of the front-right and rear-right tires 
(Eqs. (9) and (11)) or for the front-left and rear-left tires (Eqs. (10) and (12)), respectively.

By applying Newton-Euler laws to the model in Figure 1, the equations of motion can be derived to the static equilibrium positions.

For the passenger seat:

$$
\begin{aligned}
& m_{a} \ddot{Z}_{a}=-F_{a k}-F_{a c}-m_{a} g, \\
& m_{b} \ddot{Z}_{b}=-F_{b k}-F_{b c}-m_{b} g, \\
& m_{c} \ddot{Z}_{c}=-F_{c k}-F_{c c}-m_{c} g, \\
& m_{d} \ddot{Z}_{d}=-F_{d k}-F_{d c}-m_{d} g .
\end{aligned}
$$

Sprung mass:

$$
\begin{aligned}
m_{s} \ddot{Z}_{s}= & F_{a k}+F_{a c}+F_{b k}+F_{b c}+F_{c k}+F_{c c}+F_{d k} \\
& +F_{d c}-\left(F_{s f l}+F_{c f l}+F_{s f r}+F_{c f r}+F_{s r l}\right. \\
& \left.+F_{c r l}+F_{s r r}+F_{c r r}+m_{s} g\right), \\
I_{\varphi} \ddot{\varphi}= & -F_{a k}-F_{a c}+F_{b k}+F_{b c}+F_{c k}+F_{c c}-F_{d k} \\
& \left.-F_{d c}\right) f \cos \varphi+\left(-F_{s f l}-F_{c f l}+F_{s f r}+F_{c f r}\right. \\
& \left.-F_{s r l}-F_{c r l}+F_{s r r}+F_{c r r}\right) \frac{s}{2} \cos \varphi,
\end{aligned}
$$

$$
\begin{aligned}
I_{\theta} \ddot{\theta}= & \left(-F_{a k}-F_{a c}-F_{b k}-F_{b c}\right) e \cos \theta+\left(F_{c k}+F_{c c}\right. \\
& \left.+F_{d k}+F_{d c}\right) h \cos \theta+\left(F_{s f l}+F_{c f l}+F_{s f r}\right. \\
& \left.+F_{c f r}\right) a \cos \theta-\left(F_{s r l}+F_{c r l}+F_{s r r}\right. \\
& \left.+F_{c r r}\right) b \cos \theta .
\end{aligned}
$$

Front-left unsprung mass:

$$
m_{u f} \ddot{Z}_{u f l}=F_{s f l}+F_{c f l}-F_{u s f l}-F_{u c f l}-m_{u f} g .
$$

Front-right unsprung mass:

$$
m_{u f} \ddot{Z}_{u f r}=F_{s f r}+F_{c f r}-F_{u s f r}-F_{u c f r}-m_{u f} g .
$$

Rear-left unsprung mass:

$$
m_{u r} \ddot{Z}_{u r l}=F_{s r l}+F_{c r l}-F_{u s r l}-F_{u c r l}-m_{u r} g .
$$

Rear-right unsprung mass:

$$
m_{u r} \ddot{Z}_{u r r}=F_{s r r}+F_{c r r}-F_{u s r r}-F_{u c r r}-m_{u r} g .
$$

The forces related to the passenger seats in Eqs. (13)(16) are expressed as:

$$
\begin{aligned}
& F_{a k}=k_{a}\left(\Delta_{u a}-\Delta_{s a}\right), \\
& F_{a c}=c_{a} \dot{\Delta}_{u a}, \\
& F_{b k}=k_{b}\left(\Delta_{u b}-\Delta_{s b}\right), \\
& F_{b c}=c_{b} \dot{\Delta}_{u b}, \\
& F_{c k}=k_{c}\left(\Delta_{u c}-\Delta_{s c}\right), \\
& F_{c c}=c_{c} \dot{\Delta}_{u c}, \\
& F_{d k}=k_{d}\left(\Delta_{u d}-\Delta_{s d}\right), \\
& F_{d c}=c_{d} \dot{\Delta}_{u d},
\end{aligned}
$$

where:

$$
\begin{aligned}
& \Delta_{u a}=Z_{a}+f \sin \varphi+e \sin \theta-Z_{s}, \\
& \Delta_{u b}=Z_{b}-f \sin \varphi+e \sin \theta-Z_{s}, \\
& \Delta_{u c}=Z_{c}-f \sin \varphi-h \sin \theta-Z_{s}, \\
& \Delta_{u d}=Z_{d}+f \sin \varphi-h \sin \theta-Z_{s}, \\
& \Delta_{s a}=\frac{m_{a} g}{k_{a}} \\
& \Delta_{s b}=\frac{m_{b} g}{k_{b}} \\
& \Delta_{s c}=\frac{m_{c} g}{k_{c}}
\end{aligned}
$$

The applied forces to the sprung mass in Eqs. (17)-(23) can be calculated as follows:

$$
F_{s f l}=100^{\left(n_{s f-1}\right)} k_{s f} \operatorname{sgn}\left(\Delta_{u f l}-\Delta_{s f l}\right)
$$

$$
\left|\Delta_{u f l}-\Delta_{s f l}\right|^{n_{s f}},
$$

$$
F_{c f l}=c_{s f} \dot{\Delta}_{u f l}
$$

$$
F_{s f r}=100^{\left(n_{s f}-1\right)} k_{s f} \operatorname{sgn}\left(\Delta_{u f r}-\Delta_{s f r}\right)
$$

$$
\left|\Delta_{u f r}-\Delta_{s f r}\right|^{n_{s f}}
$$




$$
\begin{aligned}
F_{c f r}= & c_{s f} \dot{\Delta}_{u f r}, \\
F_{s r l}= & 100^{\left(n_{s r}-1\right)} k_{s r} \operatorname{sgn}\left(\Delta_{u r l}-\Delta_{s r l}\right) \\
& \left|\Delta_{u r l}-\Delta_{s r l}\right|^{n_{s r}}, \\
F_{c r l}= & c_{s r} \dot{\Delta}_{u r l}, \\
F_{s r r}= & 100^{\left(n_{s r}-1\right)} k_{s r} \operatorname{sgn}\left(\Delta_{u r r}-\Delta_{s r r}\right) \\
& \left|\Delta_{u r r}-\Delta_{s r r}\right|^{n_{s r}}, \\
F_{c r r}= & c_{s r} \dot{\Delta}_{u r r},
\end{aligned}
$$

where:

$$
\begin{aligned}
\Delta_{u f l}= & Z_{s}+\frac{s}{2} \sin \varphi-a \sin \theta-Z_{u f l} \\
\Delta_{u f r}= & Z_{s}-\frac{s}{2} \sin \varphi-a \sin \theta-Z_{u f r} \\
\Delta_{u r l}= & Z_{s}+\frac{s}{2} \sin \varphi+b \sin \theta-Z_{u r l} \\
\Delta_{u r r}= & Z_{s}-\frac{s}{2} \sin \varphi+b \sin \theta-Z_{u r r} \\
\Delta_{s f l}= & {\left[\frac { 1 } { 1 0 0 ^ { ( n _ { s f } - 1 ) } k _ { s f } } \left(\frac{\left(m_{s} g\right) b}{2(a+b)}+W_{a f l}+W_{b f l}\right.\right.} \\
& \left.\left.+W_{c f l}+W_{d f l}\right)\right]^{\frac{1}{n_{s f}}}
\end{aligned}
$$$$
\Delta_{s f r}=\left[\frac { 1 } { 1 0 0 ^ { ( n _ { s f } - 1 ) } k _ { s f } } \left(\frac{\left(m_{s} g\right) b}{2(a+b)}+W_{a f r}+W_{b f r}\right.\right.
$$$$
\left.\left.+W_{c f r}+W_{d f r}\right)\right]^{\frac{1}{n_{s f}}}
$$

$$
\begin{gathered}
\Delta_{s r l}=\left[\frac { 1 } { 1 0 0 ^ { ( n _ { s r } - 1 ) } k _ { s r } } \left(\frac{\left(m_{s} g\right) a}{2(a+b)}+W_{a r l}+W_{b r l}\right.\right. \\
\left.\left.+W_{c r l}+W_{d r l}\right)\right]^{\frac{1}{n_{s r}}}
\end{gathered}
$$$$
\Delta_{s r r}=\left[\frac { 1 } { 1 0 0 ^ { ( n _ { s r } - 1 ) } k _ { s r } } \left(\frac{\left(m_{s} g\right) a}{2(a+b)}+W_{a r r}+W_{b r r}\right.\right.
$$$$
\left.\left.+W_{c r r}+W_{d r r}\right)\right]^{\frac{1}{n_{s r}}}
$$

where $W_{p i j}$ is the effect of passengers weight on the sprung mass corners.

The applied forces related to the unsprung mass in Eqs. (20)-(23) are expressed as:

$$
\begin{aligned}
& F_{u s f l}= 100^{\left(n_{u s f}-1\right)} k_{u s f} \operatorname{sgn}\left(\Delta_{u s f l}-\Delta_{s u f l}\right) \\
&\left|\Delta_{u s f l}-\Delta_{s u f l}\right|^{n_{u s f}}, \\
& F_{u c f l}= c_{u s f} \dot{\Delta}_{u s f l}, \\
& F_{u s f r}= 100^{\left(n_{u s f}-1\right)} k_{u s f} \operatorname{sgn}\left(\Delta_{u s f r}-\Delta_{s u f r}\right) \\
&\left|\Delta_{u s f r}-\Delta_{s u f r}\right|^{n_{u s f}}, \\
& F_{u c f r}= c_{u s f} \dot{\Delta}_{u s f r}, \\
& F_{u s r l}= 100^{\left(n_{u s r}-1\right)} k_{u s r} \operatorname{sgn}\left(\Delta_{u s r l}-\Delta_{s u r l}\right) \\
&\left|\Delta_{u s r l}-\Delta_{s u r l}\right|^{n_{u s r}}, \\
& F_{u c r l}= c_{u s r} \dot{\Delta}_{u s r l}, \\
& F_{u s r r}= 100^{\left(n_{u s r}-1\right)} k_{u s r} \operatorname{sgn}\left(\Delta_{u s r r}-\Delta_{s u r r}\right) \\
& F_{u c r r}= c_{u s r} \dot{\Delta}_{u s r r}, \\
&\left|\Delta_{u s r r}-\Delta_{s u r r}\right|^{n_{u s r}},
\end{aligned}
$$

where:

$$
\begin{aligned}
& \Delta_{u s f l}=Z_{u s f l}-A \sin (2 \pi f t+\beta), \\
& \Delta_{u s f r}=Z_{u s f r}-A \sin (2 \pi f t), \\
& \Delta_{u s r l}=Z_{u s r l}-A \sin (2 \pi f t+\beta+\alpha), \\
& \Delta_{u s r r}=Z_{u s r r}-A \sin (2 \pi f t+\alpha),
\end{aligned}
$$

$$
\begin{aligned}
\Delta_{s u f l}= & {\left[\frac { 1 } { 1 0 0 ^ { ( n _ { u s f } - 1 ) } k _ { u s f } } \left(\frac{\left(m_{s} g\right) b}{2(a+b)}+m_{u f} g\right.\right.} \\
& \left.\left.+W_{a f l}+W_{b f l}+W_{c f l}+W_{d f l}\right)\right]^{\frac{1}{n_{u s f}}},
\end{aligned}
$$

$$
\begin{aligned}
\Delta_{s u f r}= & {\left[\frac { 1 } { 1 0 0 ^ { ( n _ { u s f } - 1 ) } k _ { u s f } } \left(\frac{\left(m_{s} g\right) b}{2(a+b)}+m_{u f} g\right.\right.} \\
& \left.\left.+W_{a f r}+W_{b f r}+W_{c f r}+W_{d f r}\right)\right]^{\frac{1}{n_{u s f}}}
\end{aligned}
$$




$$
\begin{aligned}
\Delta_{\text {surl }}= & {\left[\frac { 1 } { 1 0 0 ^ { ( n _ { u s r } - 1 ) } k _ { u s r } } \left(\frac{\left(m_{s} g\right) a}{2(a+b)}+m_{u r} g\right.\right.} \\
& \left.\left.+W_{a r l}+W_{b r l}+W_{c r l}+W_{d r l}\right)\right]^{\frac{1}{n_{u s r}}}, \\
\Delta_{\text {surr }}= & {\left[\frac { 1 } { 1 0 0 ^ { ( n _ { u s r } - 1 ) } k _ { u s r } } \left(\frac{\left(m_{s} g\right) a}{2(a+b)}+m_{u r} g\right.\right.} \\
& \left.\left.+W_{a r r}+W_{b r r}+W_{c r r}+W_{d r r}\right)\right]^{\frac{1}{n_{u s r}}}
\end{aligned}
$$

\section{Numerical results and discussion}

The numerical solution to the nonlinear differential equations governing the problem was carried out by a fourth-order Runge-Kutta numerical method provided by MATLAB. The frequency response diagram, bifurcation diagram, time series, phase plane portrait, power spectrum, Poincare' maps, and Lyapunov exponents were used to identify the chaotic response. To guarantee that the data being used are in a steady state, the first few hundred-time series data of the integration were neglected. The results of the next few hundred-time series were retained to carry out the analysis. The parameters of the vehicle model used in the numerical study are shown in Table 1.

In order to validate the developed computer codes of the numerical simulations, the obtained results of full vehicle model (with seven degrees of freedom) were first compared to those of Ref. [18]. Then, passengers were added to the model with seven degrees of freedom, and the desired changes were applied to this model. Figures 2 and 3 show the average results of the present work and those of Ref. [18] for a model with seven degrees of freedom. It can be seen that there is close agreement between the results of the present work for a model with seven degrees of freedom and the aforementioned reference.

After validation of the developed computer codes, the desired changes were applied to the model with seven degrees of freedom, and then the passengers were added to it. In order to evaluate the effect of passengers on the nonlinear behavior of the vehicle, the following steps were taken [19]:

1. One passenger was placed in the driver's position and the eight degrees of freedom of full vehicle model were created;

2. In addition to the driver, another passenger was placed on the seat to the right of the driver and the nine degrees of freedom model were created;
Table 1. Parameters for numerical solution.

\begin{tabular}{ll}
\hline \multicolumn{1}{c}{ Parameter } & \multicolumn{1}{c}{ Value } \\
\hline$m_{s}$ & $1500 \mathrm{~kg}$ \\
$m_{a}, m_{b}, m_{c}, m_{d}$ & $90 \mathrm{~kg}$ \\
$I_{\varphi}$ & $460 \mathrm{~kg} \mathrm{~m}$ \\
$I_{\theta}$ & $2160 \mathrm{~kg} \mathrm{~m}$ \\
$m_{u f}$ & $59 \mathrm{~kg}$ \\
$m_{u r}$ & $59 \mathrm{~kg}$ \\
$k_{s f}$ & $35000 \mathrm{~N} / \mathrm{m}$ \\
$k_{s r}$ & $38000 \mathrm{~N} / \mathrm{m}$ \\
$k_{a}, k_{b}, k_{c}, k_{d}$ & $10000 \mathrm{~N} / \mathrm{m}$ \\
$n_{s f}, n_{s r}$ & 1.5 \\
$c_{s u f}$ & $1000 \mathrm{Ns} / \mathrm{m}$ \\
$c_{s d f}$ & $720 \mathrm{Ns} / \mathrm{m}$ \\
$c_{s u r}$ & $1000 \mathrm{Ns} / \mathrm{m}$ \\
$c_{s d r}$ & $720 \mathrm{Ns} / \mathrm{m}$ \\
$c_{a}, c_{b}, c_{c}, c_{d}$ & $150 \mathrm{Ns} / \mathrm{m}$ \\
$k_{u s f}, k_{u s r}$ & $190000 \mathrm{~N} / \mathrm{m}$ \\
$c_{u s f}, c_{u s r}$ & $10 \mathrm{Ns} / \mathrm{m}$ \\
$n_{u s f}, n_{u s r}$ & 1.25 \\
$a$ & $1.4 \mathrm{~m}$ \\
$b$ & $1.7 \mathrm{~m}$ \\
$s$ & $3 \mathrm{~m}$ \\
$e$ & $0.35 \mathrm{~m}$ \\
$f$ & $0.37 \mathrm{~m}$ \\
$h$ & $0.40 \mathrm{~m}$ \\
\hline &
\end{tabular}

3. The third passenger was placed behind the driver's position, thus the ten degrees of freedom of full vehicle model were created;

4. The fourth passenger was added to the vehicle and the eleven degrees of freedom of full vehicle model were created.

To evaluate the effect of the above-mentioned changes on the seven degrees of freedom model, Poincaré maps were used. Figure 4 shows the Poincaré maps of the heave motion of the sprung mass and the front-left unsprung mass in the specified control parameters of the exciting force for the above cases. As shown above, by adding the passenger (mass) to the seven degrees of freedom vehicle model, scattering of the points on the Poincaré map was reduced, and the attractors would tend to change from the regular one. Hence, this work is conducted to study the nonlinear behaviors of the full vehicle model with four passengers in the following sections.

\subsection{Nonlinear frequency response analysis}

The frequency response diagram, obtained by plotting the amplitude of the oscillating system versus the 


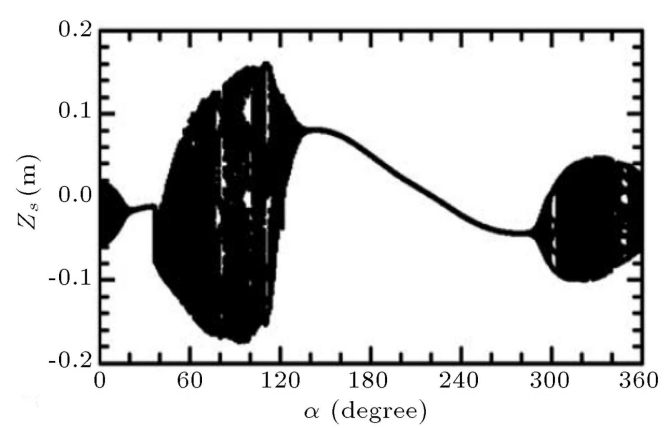

(a)

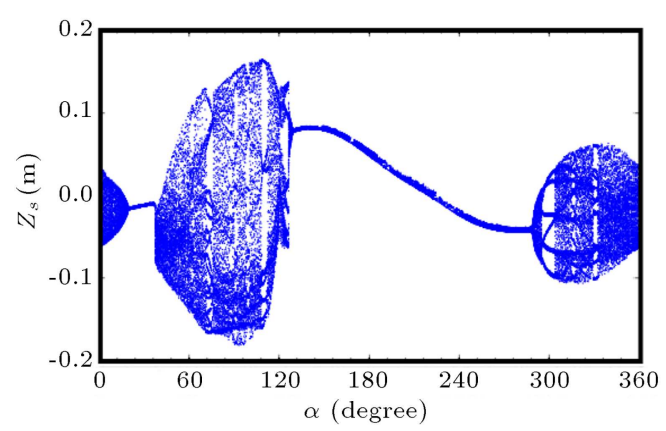

(c)

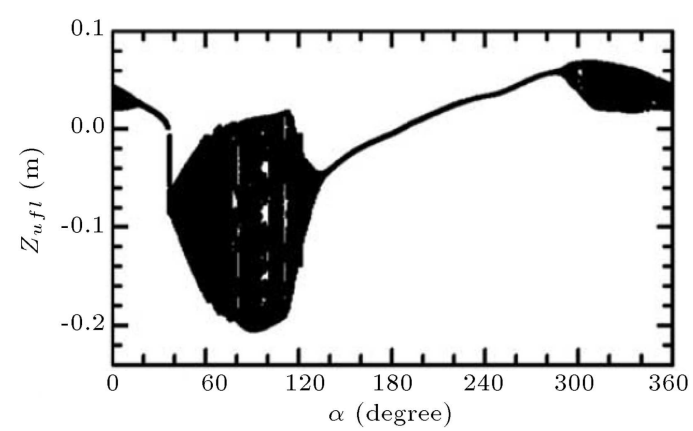

(b)

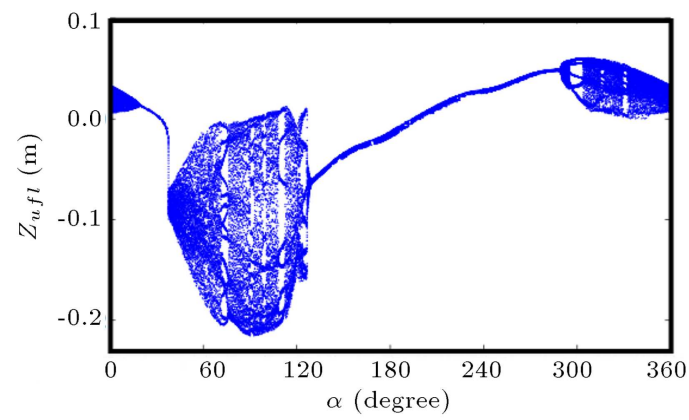

(d)

Figure 2. Bifurcation diagrams of $Z_{s}(t)$ and $Z_{u f l}(t)$ obtained by varying $\alpha\left(A=0.06 \mathrm{~m}, f=3.2 \mathrm{~Hz}, \beta=9^{\circ}\right)$ : (a) and (b) are the results of Ref. [18]; (c) and (d) are the results of this research.

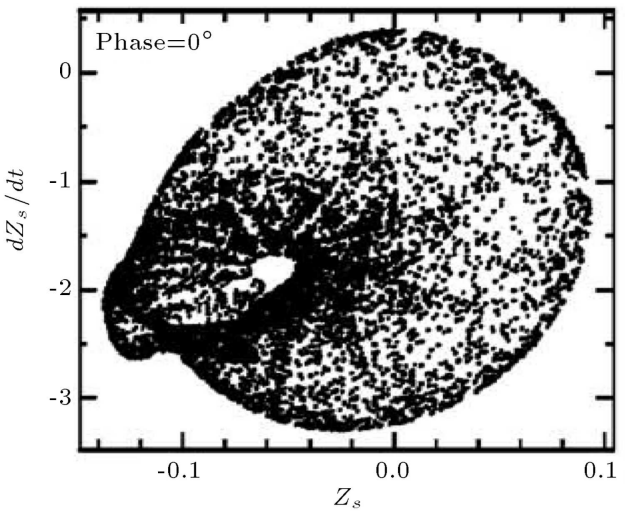

(a)

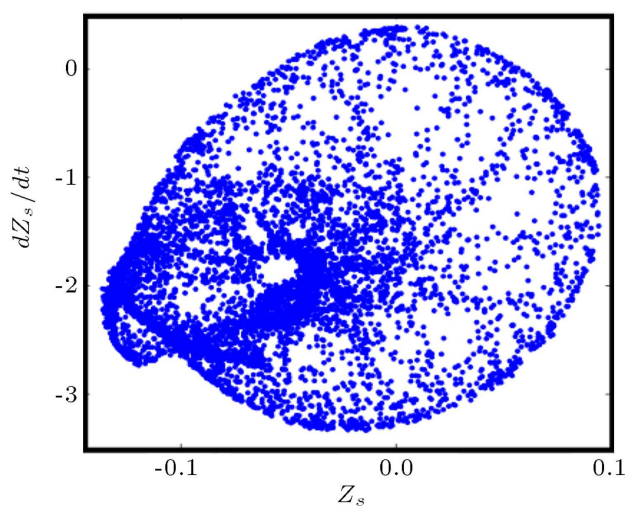

(c)

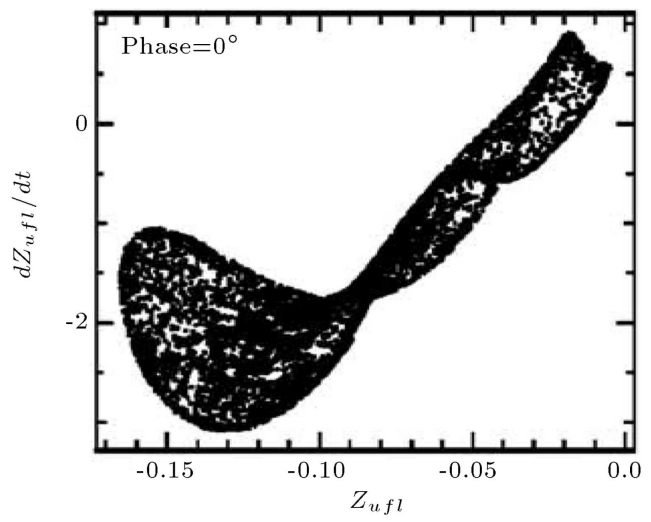

(b)

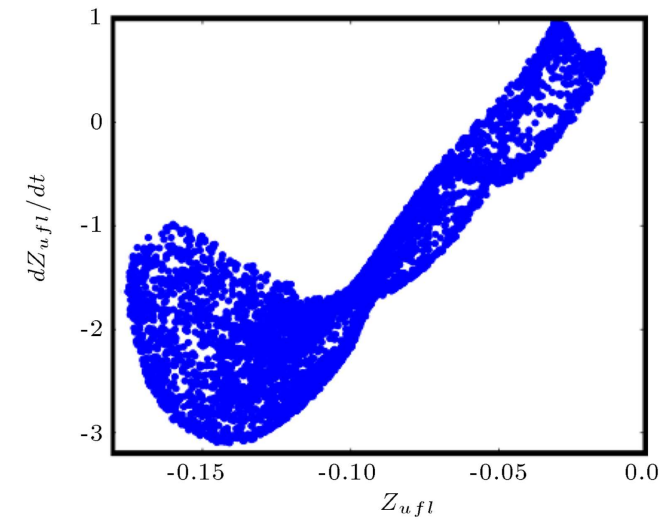

(d)

Figure 3. Poincaré maps of chaotic motion of $Z_{s}(t)$ and $Z_{u f l}(t)\left(A=0.06 \mathrm{~m}, f=3.2 \mathrm{~Hz}, \alpha=58^{\circ}, \beta=9^{\circ}\right)$ : (a) and (b) are the results of Ref. [18]; (c) and (d) are the results of this research. 


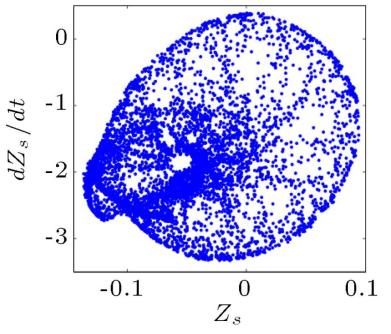

(a)

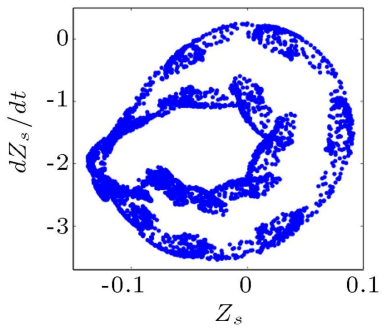

(c)

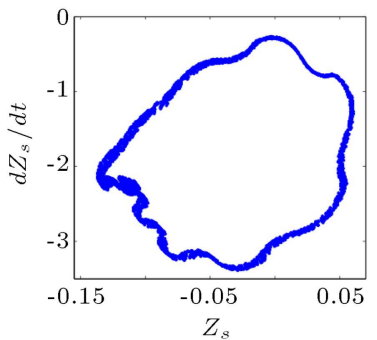

(e)

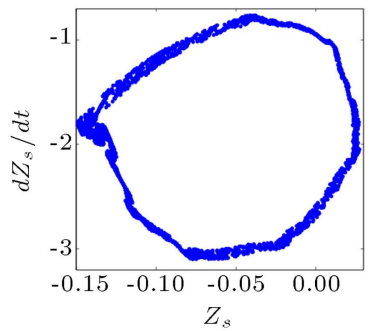

(g)

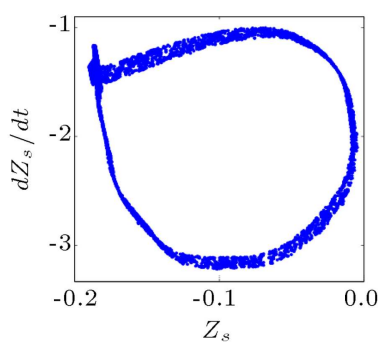

(i)

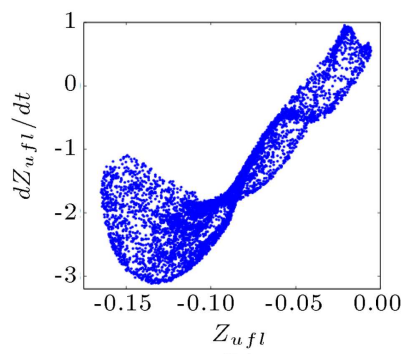

(b)

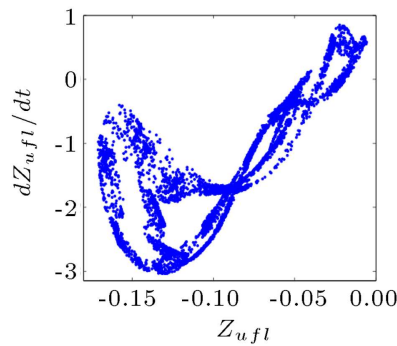

(d)

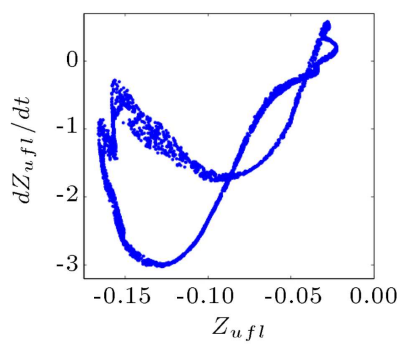

(f)

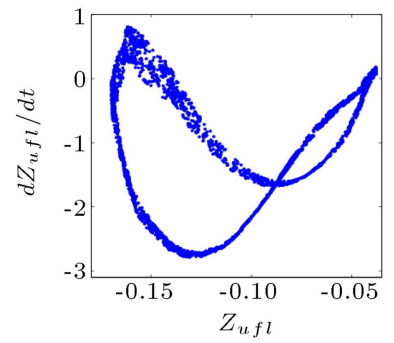

(h)

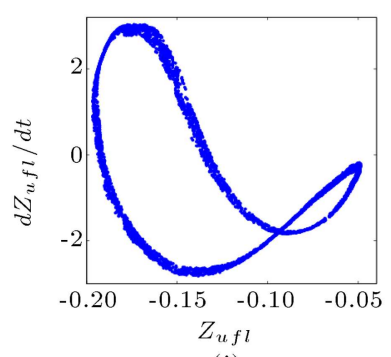

(j)
Figure 4. Poincaré maps of chaotic motion of $Z_{s}(t)$ and $Z_{u f l}(t)\left(A=0.06 \mathrm{~m}, f=3.2 \mathrm{~Hz}, \alpha=58^{\circ}, \beta=9^{\circ}\right):(\mathrm{a})$ and (b) seven-d.o.f vehicle; (c) and (d) eight-d.o.f vehicle; (e) and (f) nine-d.o.f vehicle; (g) and (h) ten-d.o.f vehicle; and (i) and (j) eleven-d.o.f vehicle.

frequency of the excitation, is often used to analyze the dynamic behavior of a system [21]. For the studied system, the frequency response diagram was calculated numerically. The amplitude was defined as an absolute value of the displacement, and the control parameter was defined as the forcing frequency of the excitation from the road surface. Since chaotic responses are possible when the forcing frequency is within an unstable region $[22,23]$, the forcing frequency for inducing chaos can be predicted by studying unstable regions in the frequency response diagrams [18]. Figure 5 shows the frequency response of the heave motion of seats (b) and (c), the heave motion of the sprung mass, and the heave motion of the unsprung mass in the front-left corner, when forcing frequency $f$ is slowly increased from 0.01 to $10 \mathrm{~Hz}$. The frequency response diagrams of the two other seats have the same characteristics. The step size of the control parameter is $\Delta_{f}=0.001 \mathrm{~Hz}$ for all of the frequency response diagrams and the time interval.

Figure 5(a) and (b) show the frequency responses of the heave motion of seats (b) and (c), respectively. There is a large jump at $f=1.4 \mathrm{~Hz}$, a slight jump at $f=3.1 \mathrm{~Hz}$, and a smaller one at $f=4 \mathrm{~Hz}$ when the frequency increases from 0.01 to $10 \mathrm{~Hz}$. These diagrams show two bifurcation regions at $4.8<f<5.14 \mathrm{~Hz}$ and $5.8<f<6.45 \mathrm{~Hz}$. Also, the results indicate unstable regions between $3.1<f<4.42 \mathrm{~Hz}$ and $5.48<f<5.62 \mathrm{~Hz}$.

The frequency responses for the heave motion of the sprung mass and the front-left unsprung mass are illustrated in Figure 5(c) and (d), respectively. In these diagrams, two jumps can be observed. A large jump at $f=3.1 \mathrm{~Hz}$ and a smaller jump at $f=4 \mathrm{~Hz}$. The bifurcation regions in the frequency responses for the heave motion of the sprung mass and the front-left unsprung mass are similar to the heave motion of the seats (a) and (b), but the jump regions are different.

The main benefit of these diagrams is the detection of jump points and unstable regions. Then, those susceptible regions can be analyzed for chaotic behavior in greater detail. Hence, frequency region $3.1<f<4.42 \mathrm{~Hz}$, as the first unstable region, which is located near the large jump in the system, has a great importance. Therefore, the dynamic behavior of the system is investigated according to Ref. [18], excitation force frequency, $f=3.2 \mathrm{~Hz}$, chosen as a constant parameter, and any change in the other parameters of excitation force. The results show that it is possible for the behavior of the system to be chaotic for some initial conditions and system characteristic parameters when the forcing frequency is near or in the unstable regions or near a jump. Here, it should be noted that frequency $f=3.2 \mathrm{~Hz}$ is the first fundamental frequency of the system of which further details will follow in the next sections.

\subsection{Bifurcation}

In this section, the effect of changing system parameters on bifurcation behavior and system responses is discussed. To generate the bifurcation diagram, the 


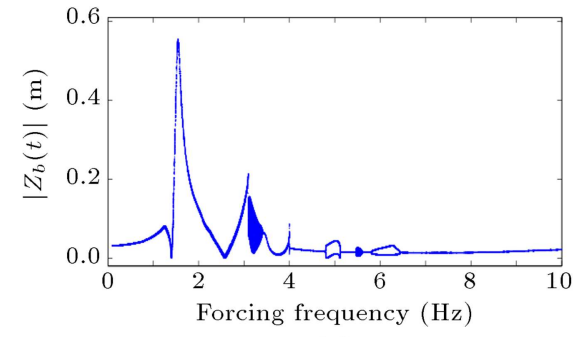

(a)

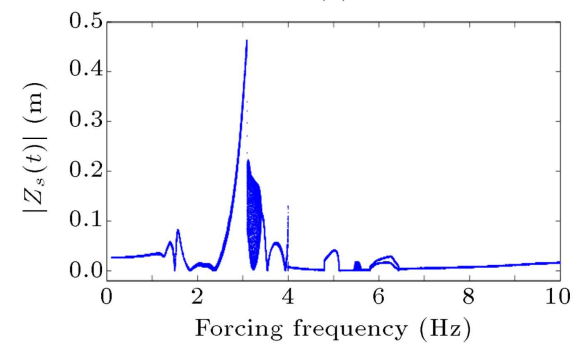

(c)

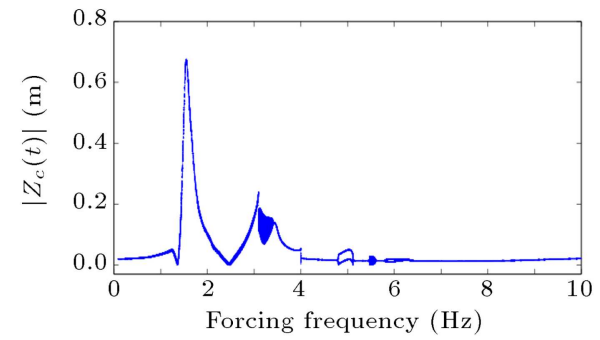

(b)

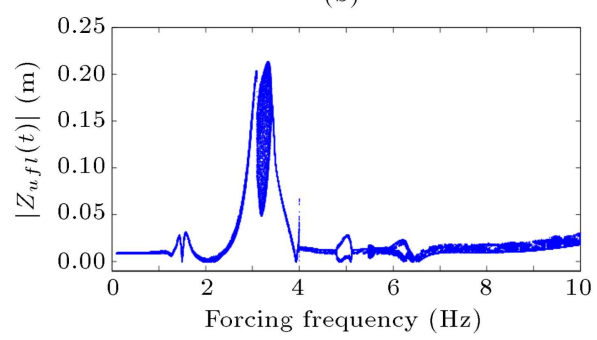

(d)

Figure 5. Frequency response diagrams by varying forcing frequency $f\left(A=0.06 \mathrm{~m}, \alpha=35^{\circ}, \beta=9^{\circ}\right):(\mathrm{a})\left|Z_{b}(t)\right|,(\mathrm{b})$ $\left|Z_{c}(t)\right|,(\mathrm{c})\left|Z_{s}(t)\right|$, and $(\mathrm{d})\left|Z_{u f l}(t)\right|$.

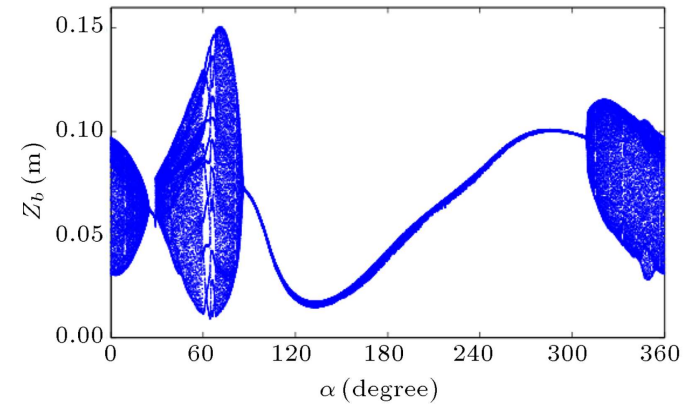

(a)

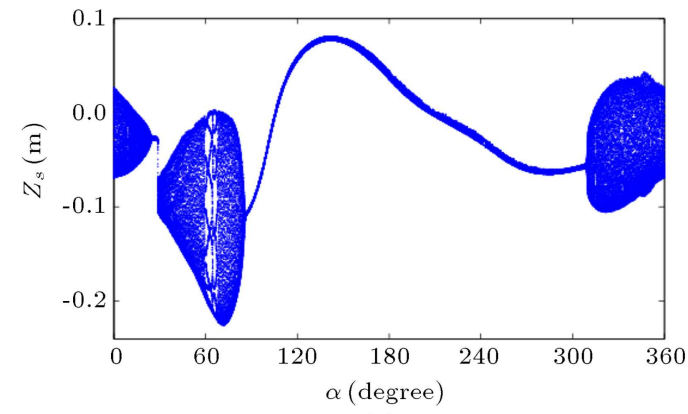

(c)

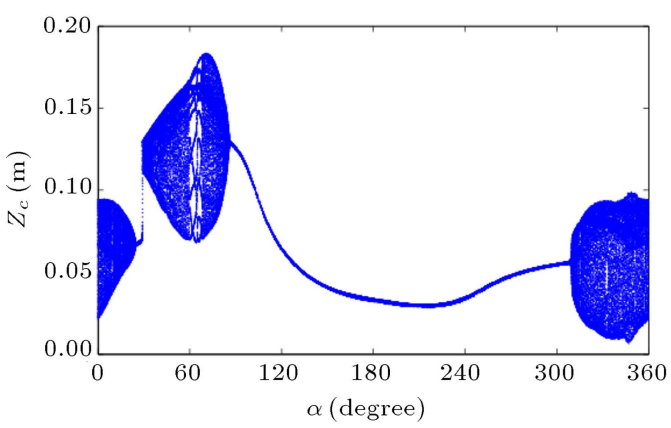

(b)

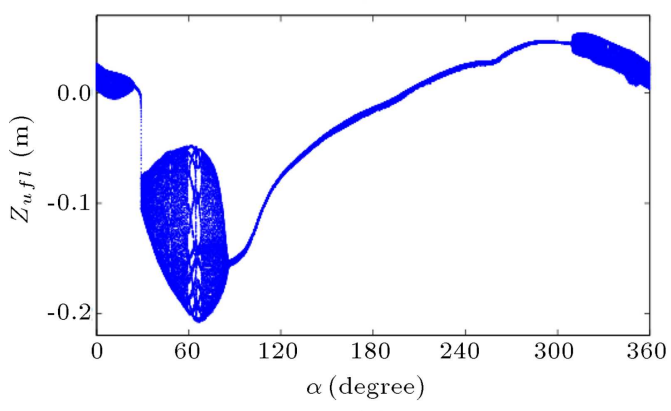

(d)

Figure 6. Bifurcation diagrams obtained by varying $\alpha\left(A=0.06 \mathrm{~m}, f=3.2 \mathrm{~Hz}, \beta=9^{\circ}\right)$ : (a) $Z_{b}(t),(\mathrm{b}) Z_{c}(t),(\mathrm{c}) Z_{s}(t)$, and (d) $Z_{u f l}(t)$.

system control parameter is varied with a fixed step, and the stated variables at the end of each step are used as the initial conditions for the next step. These data points are then plotted versus the system control parameters. If the motion is regular (periodic) at the specific control parameter, the bifurcation diagram should contain a finite number of separate points. When the motion is quasi-periodic or chaotic, the data points in the bifurcation diagram are distributed along a line.

In this study, the effect of time delays $\alpha$ and $\beta$ on the appearance of chaos is discussed. The bifurcation diagrams in $0^{\circ} \leq \alpha, \beta \leq 360^{\circ}$ were constructed via numerical integration of Eq. (13)-(23), and the initial conditions were set to zero. The results are presented in Figures 6 to 8. As shown in Figure 6, the dynamic behaviors of seats (b) and (c), sprung mass, and frontleft unsprung mass are irregular when time delay $\alpha$ is in regions $0^{\circ} \leq \alpha \leq 25^{\circ}, 28^{\circ} \leq \alpha \leq 86^{\circ}$, and $310^{\circ} \leq \alpha \leq 360^{\circ}$, and is regular with period-one (1T) and irregular with small amplitude between these 


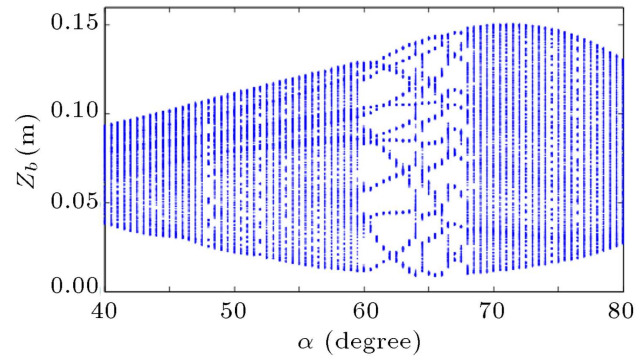

(a)

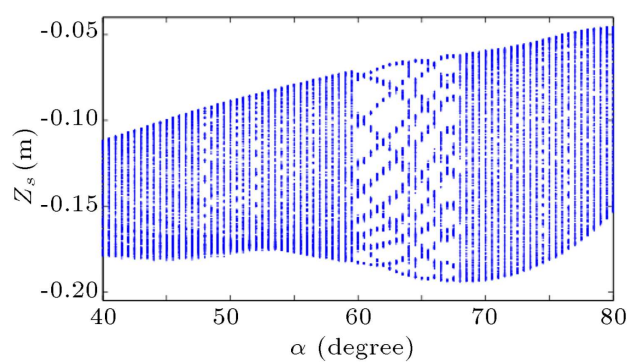

(c)

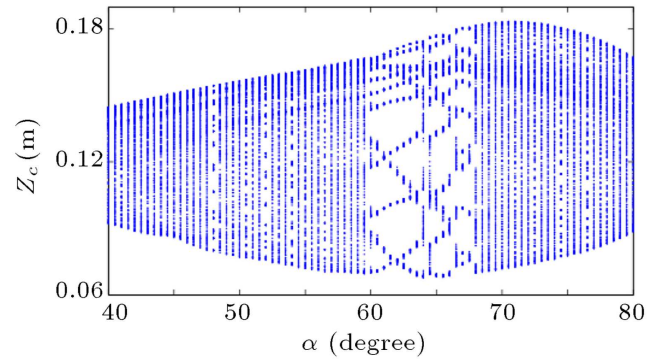

(b)

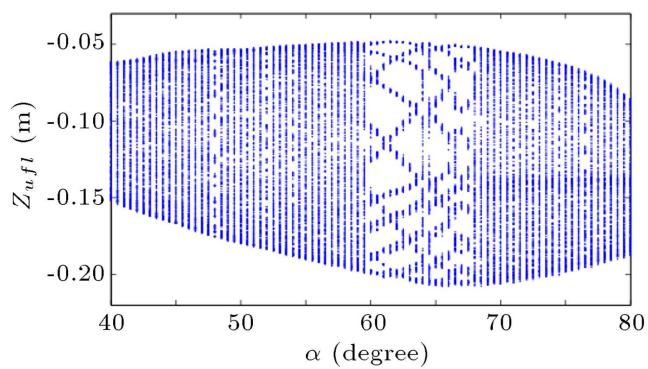

(d)

Figure 7. Zoomed section of Figure 6 between $\alpha=40^{\circ}$ and $\alpha=80^{\circ}$.

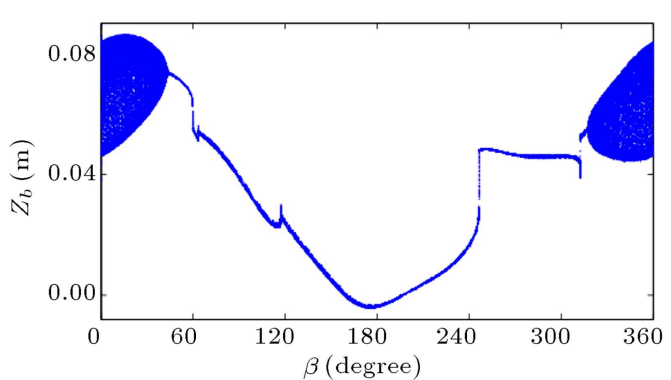

(a)

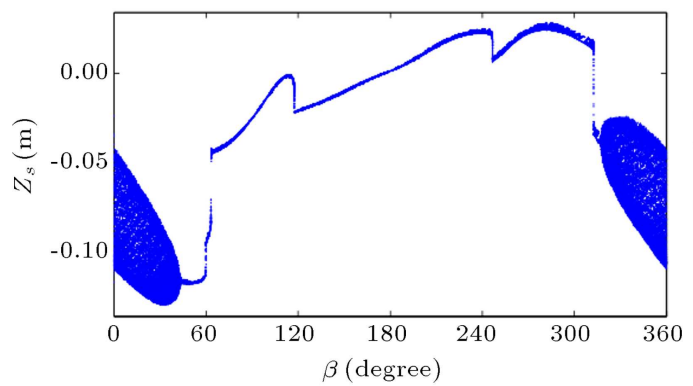

(c)

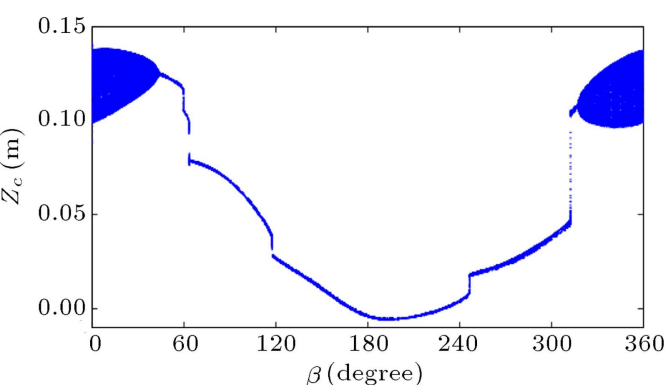

(b)

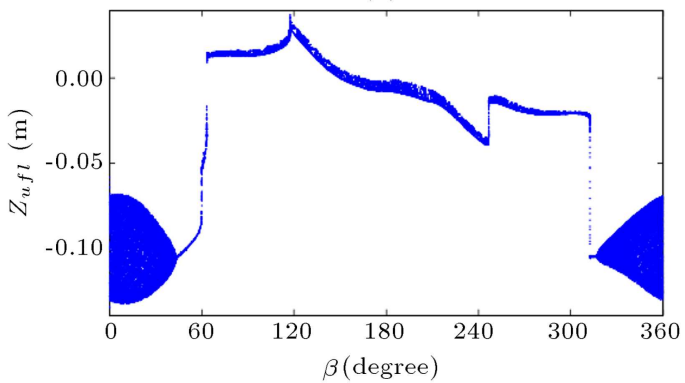

(d)

Figure 8. Bifurcation diagrams obtained by varying $\beta\left(A=0.06 \mathrm{~m}, f=3.2 \mathrm{~Hz}, \alpha=35^{\circ}\right)$ : (a) $Z_{b}(t),(\mathrm{b}) Z_{c}(t),(\mathrm{c}) Z_{s}(t)$, and (d) $Z_{u f l}(t)$.

regions. Figure 7 shows the zoomed section of the previous figure for better visualization. As shown in Figure 7, the motion of seats (b) and (c), sprung mass, and front-left unsprung mass becomes sub-harmonic with period $(12 \mathrm{~T})$ at $60^{\circ} \leq \alpha \leq 68^{\circ}$. These figures also indicate that the responses of seats (b) and (c), sprung mass, and front-left unsprung mass become chaotic and regular at the same time. Since the regular behavior of the system without period doubling changed to an irregular one, this type of bifurcation is considered as a dangerous bifurcation [24].
In Figure 8, the control parameter is $\beta$; the bifurcation diagrams show that the irregular behavior in the dynamic response of seats (b) and (c), sprung mass, and front-left unsprung mass has two rather large regions: $0^{\circ} \leq \beta \leq 44^{\circ}$ and $316^{\circ} \leq \beta \leq$ $360^{\circ}$. It is observed that the bifurcation in this case is also a dangerous one [24]. The periodic motion (1T) and irregular motion with small amplitude can be detected in the dynamic behavior of system between these two regions. The dynamic behavior of the other components of the system is almost similar when the 
time delays $\alpha$ and $\beta$ are changed in the domain of $0^{\circ} \leq \alpha, \beta \leq 360^{\circ}$.

\subsection{Chaos}

The bifurcation diagrams are used to detect the dynamic behavior of system and identify the irregular regions by changing the control parameters. With detailed analysis of system behavior and confirmation of the chaotic responses, there is the need for other identifying techniques. Therefore, time series, phase plane portrait, power spectrum, Poincaré map, and Lyapunov exponents are used to identify and analyze the chaotic responses. Also, the autocorrelation function is employed to investigate the correlation between signals or trajectories $x(t)$ and $x(t+T)$, where $T$ is time delay [24].

Figures 9 and 10 show the time series, phase plane portrait, power spectrum, and Poincaré map for the heave motion of the driver's seat and sprung mass at $\alpha=35^{\circ}$ and $\beta=9^{\circ}$, respectively. As shown, the period of motion is not detectable in time series, and the phase plane portraits are the trajectories that are repeated and tend to fill up a portion of the phase space. The power spectrum diagrams are continuous or broad-band spectra, and the Poincaré maps consist of a pile of points in the phase space or have a fractal structure, which confirms the chaotic motion at these control parameters.

Figures 11 and 12 show the time series, phase plane portrait, power spectrum, and Poincaré map

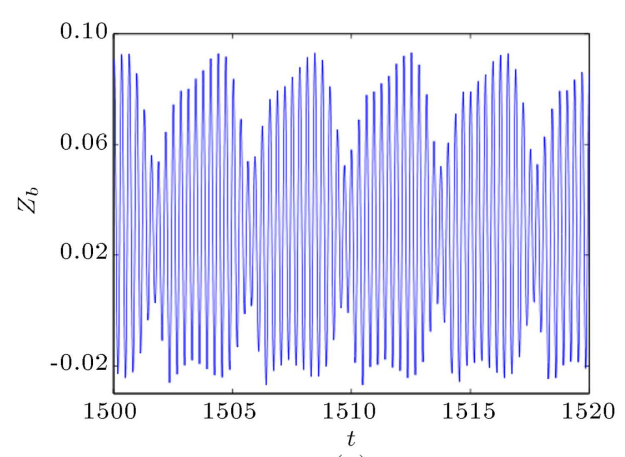

(a)

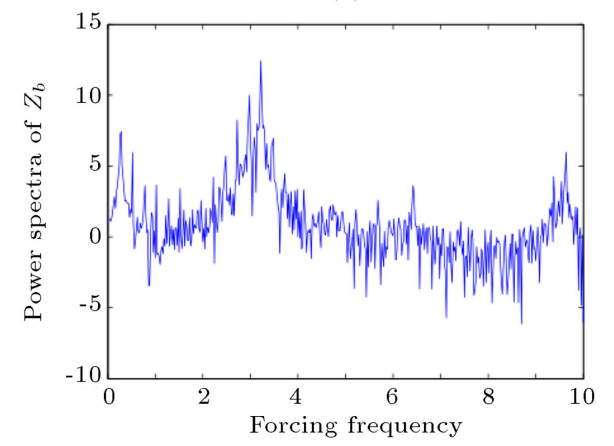

(c) of the driver's seat and sprung mass at $\alpha=66^{\circ}$, respectively. The dynamic response of the system is sub-harmonic with period (12T). As shown in Figure $11(\mathrm{~b})$, the phase plane portrait is closed after 12 routes, the power spectrum has a clear peak at the frequencies $3.2 / 12$, and there are twelve discrete points in the Poincaré map that confirm the sub-harmonic (period-12) motion in the driver's seat. Similarly, as shown in Figure 12, the dynamic response of the sprung mass demonstrates the sub-harmonic motion of period $(12 \mathrm{~T})$ at $\alpha=66^{\circ}$. As mentioned before (Section 3.1), frequency $f=3.2 \mathrm{~Hz}$ is the first fundamental frequency. As shown in Figures 11(c) and $12(\mathrm{c})$, there is a clear peak in $f=3.2 \mathrm{~Hz}$, and other sub-harmonics are a fraction of this frequency. Since the power content of this frequency is very high, the dynamic behavior of the system at this frequency has great importance.

Another important technique for identifying chaotic motion is the Lyapanov exponents. The Lyapunov exponents determine the average rate of the exponential expansion or contraction in the direction of an initial deviation $y(0)$ on a trajectory of the system, which is given by:

$$
\bar{\lambda}_{i}=\lim _{t \rightarrow \infty} \frac{1}{t} \ln \left(\frac{\|y(t)\|}{\|y(0)\|}\right),
$$

where symbol \|\| denotes a vector norm and $\bar{\lambda}_{i}$ is called the Lyapunov exponent. If the maximum

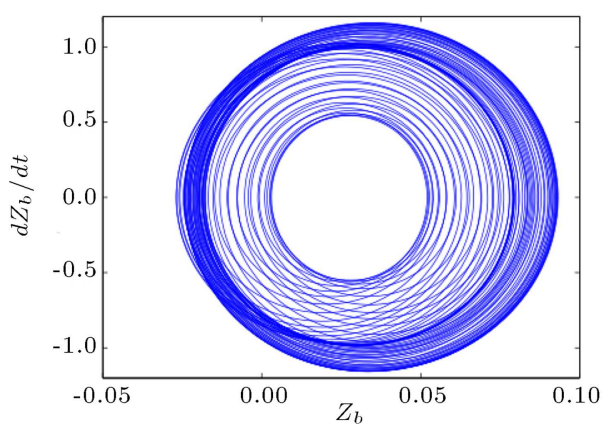

(b)

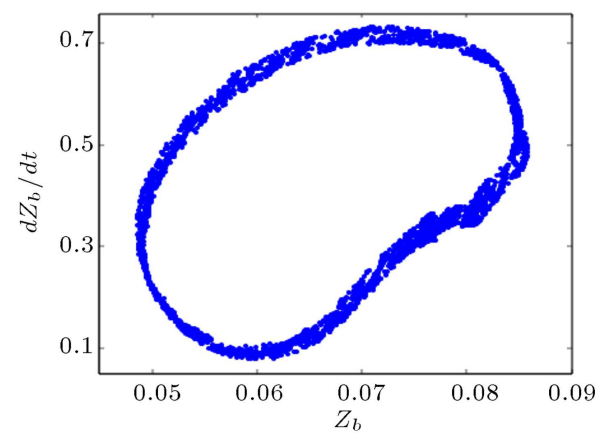

(d)

Figure 9. (a) Time series, (b) Phase plane portrait, (c) power spectrum, and (d) Poincaré map of chaotic motion of $Z_{b}(t)$ $\left(A=0.06 \mathrm{~m}, f=3.2 \mathrm{~Hz}, \alpha=35^{\circ}, \beta=9^{\circ}\right)$. 


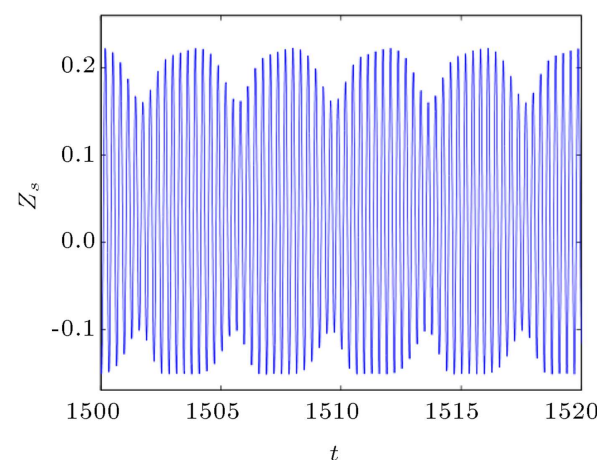

(a)

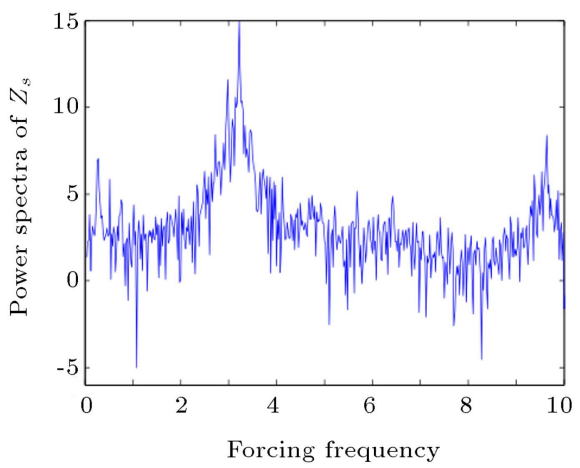

(c)

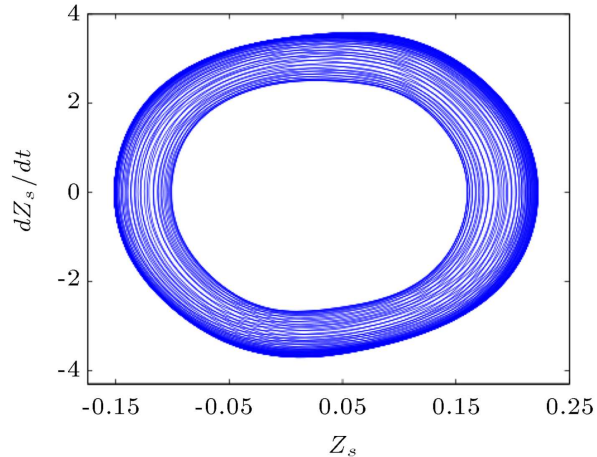

(b)

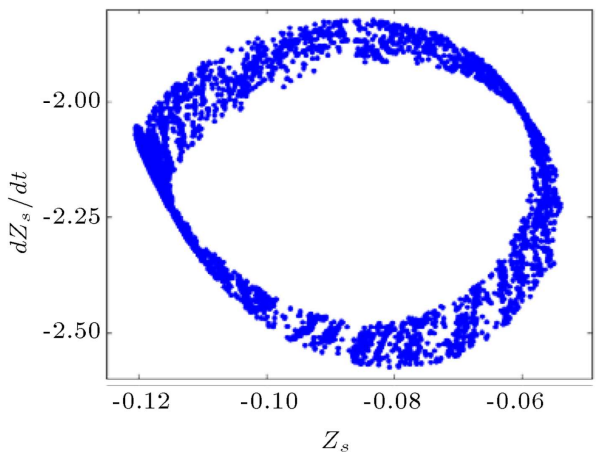

(d)

Figure 10. (a) Time series, (b) phase plane portrait, (c) power spectrum, and (d) Poincaré map of chaotic motion of $Z_{s}(t)\left(A=0.06 \mathrm{~m}, f=3.2 \mathrm{~Hz}, \alpha=35^{\circ}, \beta=9^{\circ}\right)$.

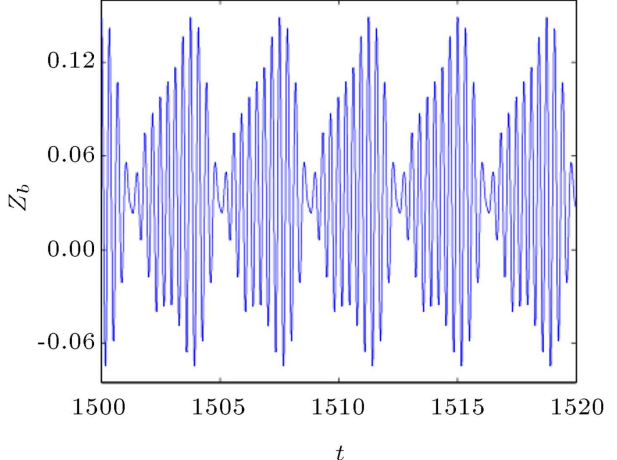

(a)

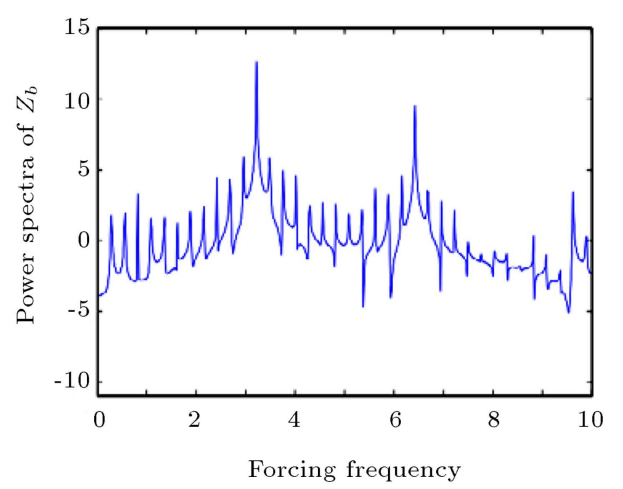

(c)

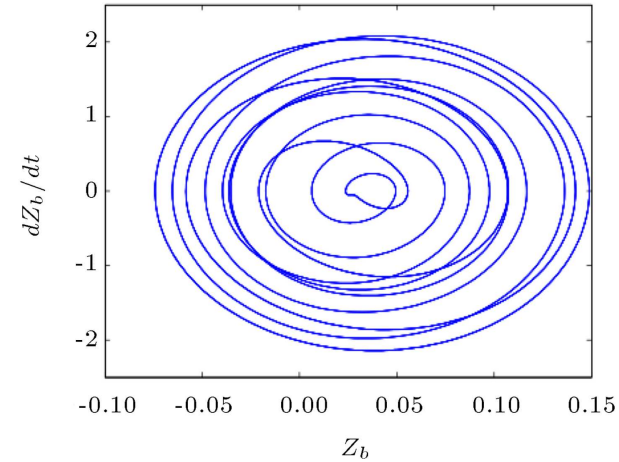

(b)

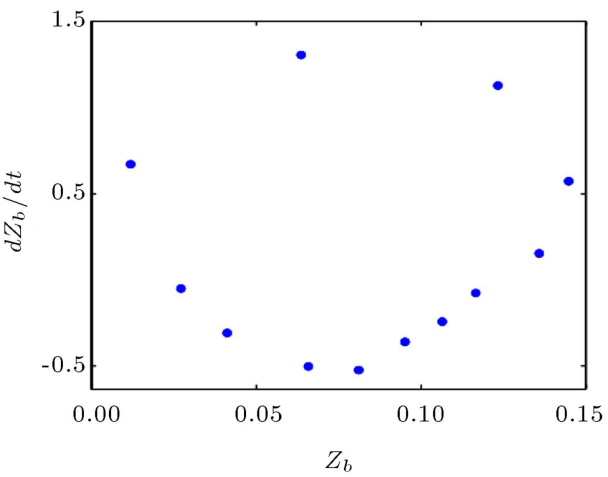

(d)

Figure 11. (a) Time series, (b) phase plane portrait, (c) power spectrum, and (d) Poincaré map of $Z_{b}(t)$ at $\alpha=66^{\circ}$. 


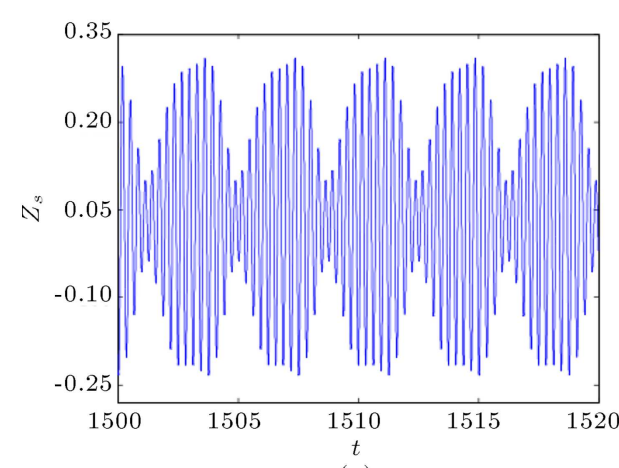

(a)

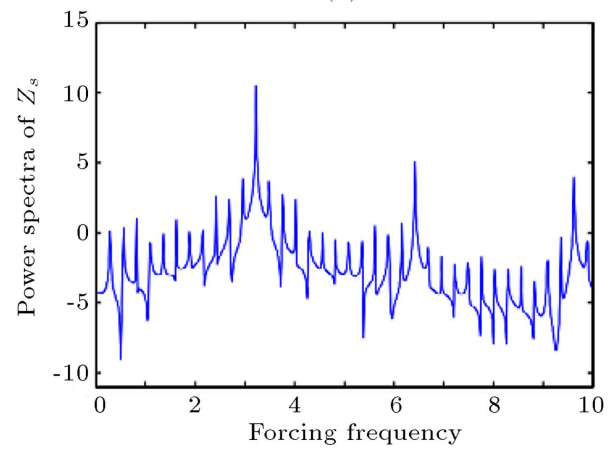

(c)

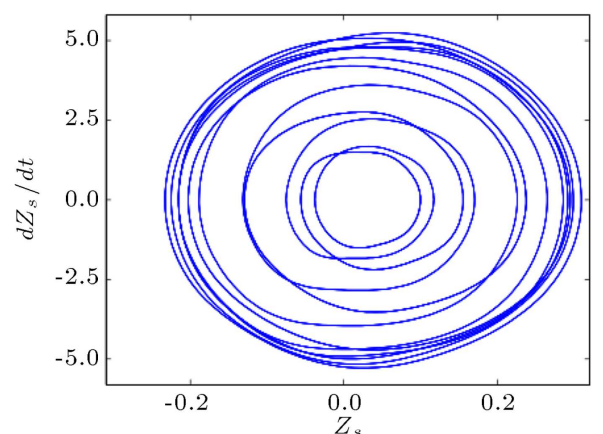

(b)

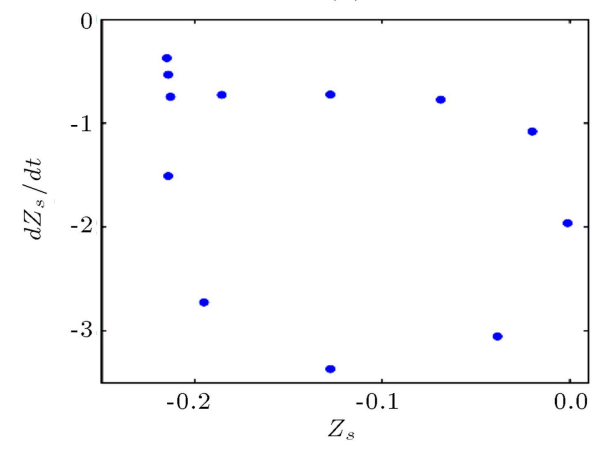

(d)

Figure 12. (a) Time series, (b) phase plane portrait, (c) power spectrum, and (d) Poincaré map of $Z_{s}(t)$ at $\alpha=66^{\circ}$.

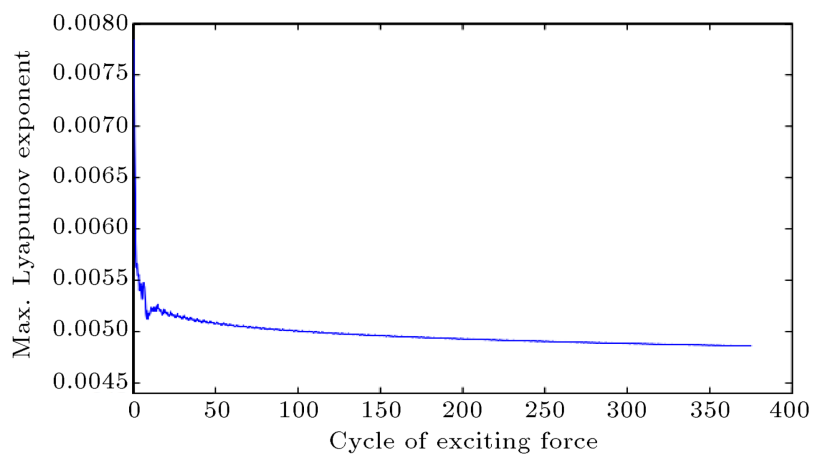

Figure 13. The maximum Lyapunov exponents $\left(A=0.06 \mathrm{~m}, f=3.2 \mathrm{~Hz}, \alpha=35^{\circ}, \beta=9^{\circ}\right)$.

Lyapunov exponent is negative or zero, there is a regular motion, and a positive maximum Lyapunov exponent will confirm the chaotic motion [24,25]. The Lyapunov exponents of system equations of motion are obtained using the algorithm given in [26]. As shown in Figure 13, positivity of maximum Lyapunov exponents at $\alpha=35^{\circ}$ and $\beta=9^{\circ}$ confirms the chaotic motion at the given control parameters.

In this section, autocorrelation function $\left(R_{x x}\right)$ is employed. Figure 14 illustrates the autocorrelation function of system's degrees of freedom. As shown in Figure 14, in the chaos state, the autocorrelation functions of heave motions of driver's seat and sprung mass (and other system's degrees of freedom) drop to zero, which proves the accuracy of the results.

By using the concept of nonlinear normal modes, the most effective modes of the system in chaotic and regular motions are investigated. Figure 15 represents the time series of nonlinear normal mode motions of vehicle body at $\alpha=35^{\circ}$, and $\beta=9^{\circ}$ for chaos state, and $\alpha=27^{\circ}$, and $\beta=9^{\circ}$ for regular state. As shown, the heave motion amplitude of body in the chaotic motion is larger than that of regular motion, and pitch motion angle is dominant compared to roll motion angle. Also, in regular motion, the pitch motion angle is dominant too. So, the results show that the effects of heave and pitch motion modes of sprung mass are greatest on the chaos state, and the smallest effect is related to roll motion.

According to the analysis provided in Sections 3.1 to 3.3 , chaotic motion in the vehicle occurs for the regions of excitation force parameters (frequency and time delay), and these regions are identified by the frequency responses and the bifurcation diagrams. However, the chaotic behavior has an oscillatory nature with unpredictable amplitudes that can lead to cyclic stresses and reduction in the life of the structures. In continuation, this idea follows that by changing important physical parameters of the system, one can change the behavior of the vehicle motions from chaotic to regular.

\section{Effect of system parameters on chaos control}

As discussed in the previous sections and shown in 


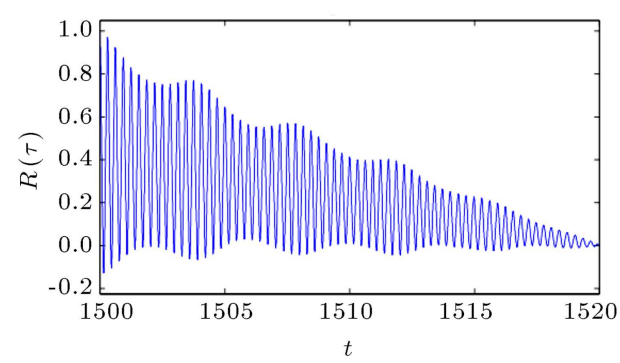

(a)

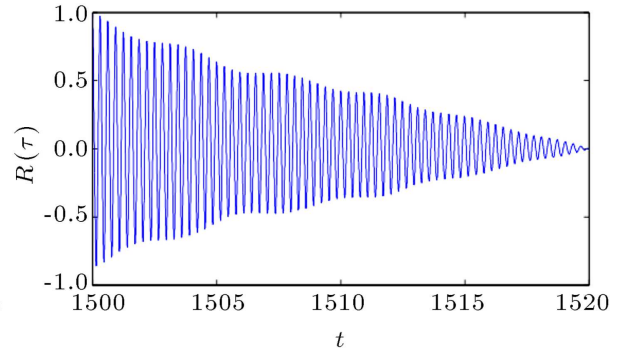

(b)

Figure 14. Autocorrelation function for chaotic motion: (a) $Z_{b}(t)$, and (b) $Z_{s}(t)$.

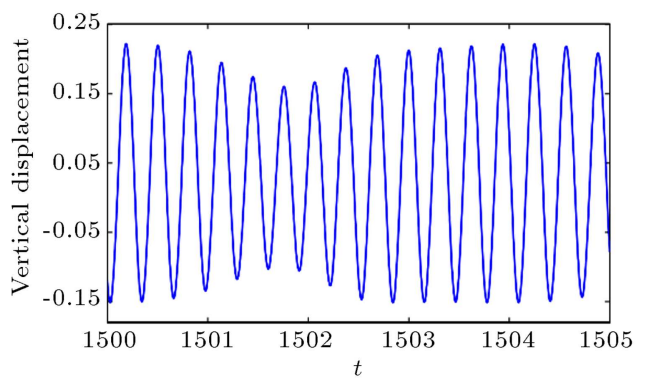

(a)

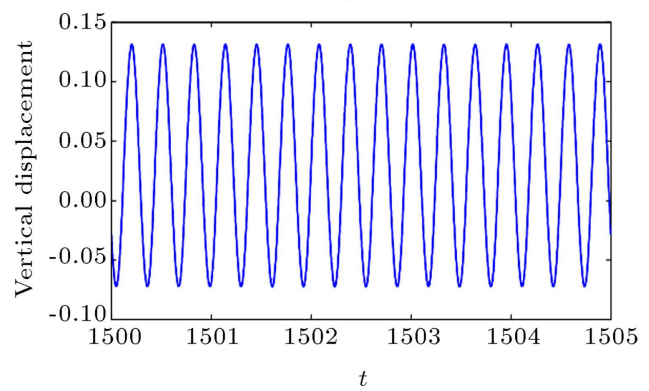

(c)

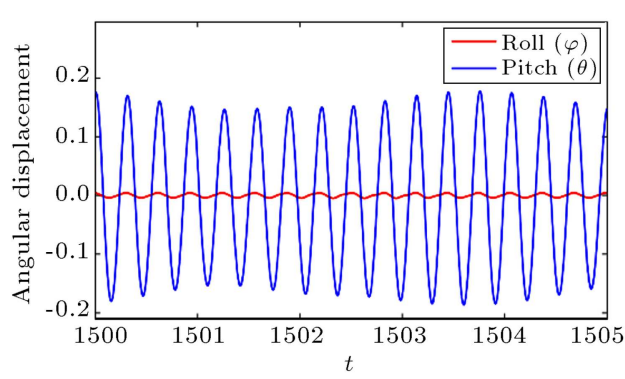

(b)

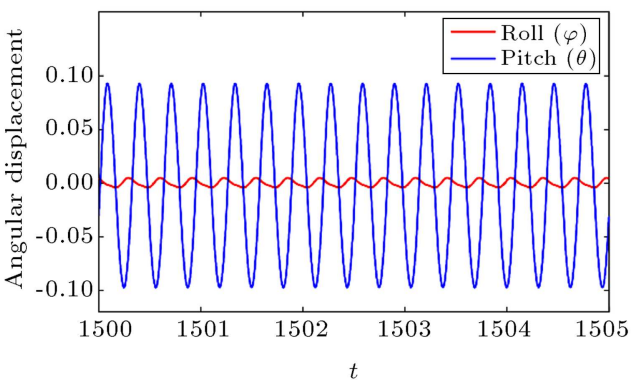

(d)

Figure 15. Time series of nonlinear normal mode motions of sprung mass: (a) and (b) are chaos status at $\alpha=35^{\circ}$ and $\beta=9^{\circ} ;(\mathrm{c})$ and $(\mathrm{d})$ are regular stutas at $\alpha=27^{\circ}$ and $\beta=9^{\circ}$.

the diagrams, chaos can be observed in a specific range of independent variables (e.g., $\alpha$ and $\beta$ ). In this section, the effects of other system parameters that may lead to removing the system from chaotic behavior are examined. In this regard, new control parameters, in place of $\alpha$ or $\beta$, are selected, and appropriate limits for these parameters are determined by means of the bifurcation diagrams. Also, it is assumed that parameters $\alpha$ and $\beta$ are fixed, and the numerical values of these parameters are such that motion of the system has been chaotic. The control parameters are: the spring stiffness of driver's seat $\left(k_{b}\right)$, the damping coefficient of driver's seat $\left(c_{b}\right)$, and the spring stiffness of suspension $\left(k_{s f}\right)$.

Figure 16 shows the effect of driver's seat spring stiffness $\left(k_{b}\right)$ on system behavior. As illustrated in the previous sections, the system response was chaotic for $f=3.2 \mathrm{~Hz}, \alpha=35^{\circ}, \beta=9^{\circ}$, and $k_{b}=10000 \mathrm{~N} / \mathrm{m}$. Figure 16 demonstrates that by choosing spring stiffness $\left(k_{b}\right)$ in the range of $22500 \mathrm{~N} / \mathrm{m}$ to $55000 \mathrm{~N} / \mathrm{m}$, the chaotic response for $f=3.2 \mathrm{~Hz}$ can be eliminated. This is a simple way to avoid chaotic behavior in the system response. Figure 17 illustrates the bifurcation phenomenon by varying the damping coefficient of driver's seat $\left(c_{b}\right)$ for driver and sprung mass. According to this figure, by choosing a damping coefficient for the driver's seat of greater than $540 \mathrm{Ns} / \mathrm{m}$, the system can perform with the regular motion. This result can be useful for changing chaotic behavior or minimizing vibration in the system. It should be noted that increasing $c_{b}$ causes the connection between the seat and sprung mass to change to a rigid connection, and this will transfer a high percentage of frame vibration to the seat. The effect of suspension spring stiffness $\left(k_{s f}\right)$ as a control parameter on system behavior is shown in Figure 18. In this case, the behavior of the driver and the sprung mass (and other parts of the system) are regular when $k_{s f}$ is less than $31,000 \mathrm{~N} / \mathrm{m}$ and more than $47,000 \mathrm{~N} / \mathrm{m}$. Detecting the irregular and regular regions of the dynamic behaviors of the vehicle 


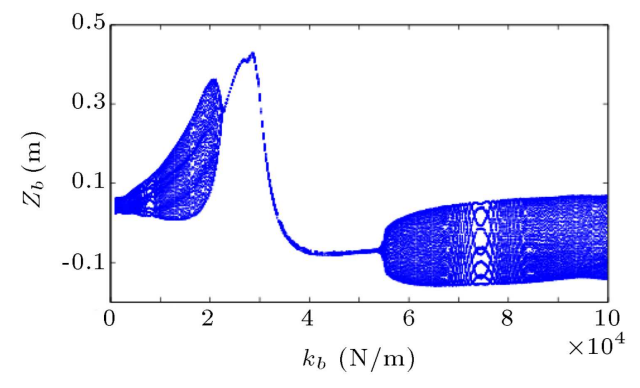

(a)

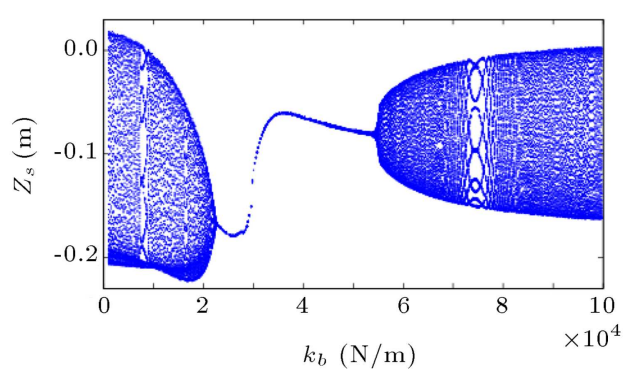

(b)

Figure 16. Bifurcation diagrams obtained by varying $k_{b}\left(A=0.06 \mathrm{~m}, f=3.2 \mathrm{~Hz}, \beta=9^{\circ}, \alpha=35^{\circ}\right)$ : (a) $Z_{b}(t)$, and (b) $Z_{s}(t)$.

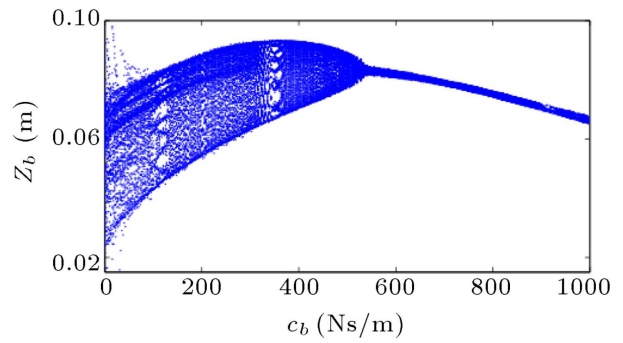

(a)

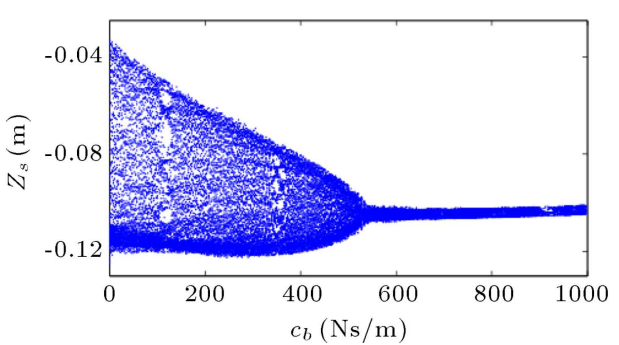

(b)

Figure 17. Bifurcation diagrams obtained by varying $c_{b}\left(A=0.06 \mathrm{~m}, f=3.2 \mathrm{~Hz}, \beta=9^{\circ}, \alpha=35^{\circ}\right):(\mathrm{a}) Z_{b}(t)$, and $(\mathrm{b})$ $Z_{s}(t)$.

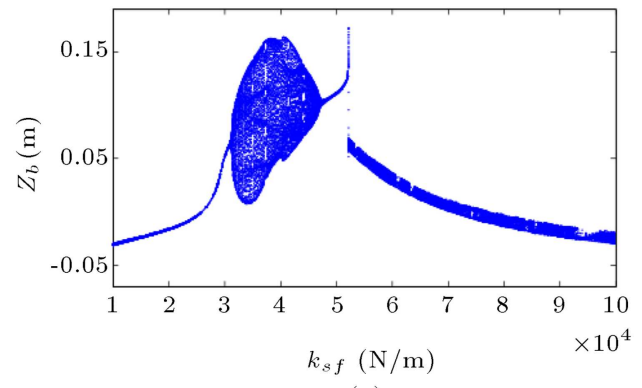

(a)

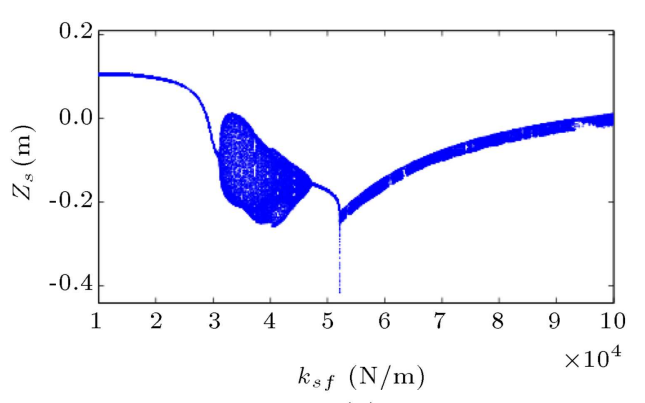

(b)

Figure 18. Bifurcation diagrams obtained by varying $k_{s f}\left(A=0.06 \mathrm{~m}, f=3.2 \mathrm{~Hz}, \beta=9^{\circ}, \alpha=35^{\circ}\right):(\mathrm{a}) Z_{b}(t)$, and $(\mathrm{b})$ $Z_{s}(t)$.

oscillating system by changing the main parameters $\left(k_{b}, c_{b}\right.$, and $\left.k_{s f}\right)$ can give insight into the designers when choosing the correct coefficients.

After determining the areas of the system parameters in which it is appropriate to return the chaotic motions to regular ones through the use of the bifurcation diagrams, the other techniques, such as the phase plane portrait and the power spectrum diagrams, are used to confirm the obtained results. Figure 19 indicates the phase plane portrait and the power spectrum diagrams for the driver's seat and sprung mass in $k_{b}=25000 \mathrm{~N} / \mathrm{m}$ and $k_{s f}=50000 \mathrm{~N} / \mathrm{m}$. As shown, the dynamic response of the system demonstrates regular motion. The closed path with a repeating pattern in the phase plane portrait, a clear peak at the frequencies 3.2 , and its integer coefficients in the power spectrum represent regular behavior at these stiffness values.

\section{Conclusion}

In this paper, the chaotic vibrations of a full-vehicle system with passengers subjected to sinusoid road disturbance with a time delay have been studied. The regions susceptible to chaotic behaviors were identified by the frequency responses. The equations of motion solved via numerical methods and the chaotic behaviors were studied in greater detail with techniques such as bifurcation diagrams, time series, phase plane portrait, power spectrum, Poincaré map, and Lyapunov exponents. The results obtained showed that the dynamic responses of the vehicle and passengers are sensitive to the road roughness amplitude, the excitation frequency (vehicle velocity), and the time delays between tires, e.g. for a road roughness of $A=0.06 \mathrm{~m}$, a frequency of $f=3.2 \mathrm{~Hz}$, and time delays of $\alpha=35^{\circ}$ and $\beta=9^{\circ}$, so the system oscillates chaotically. When 


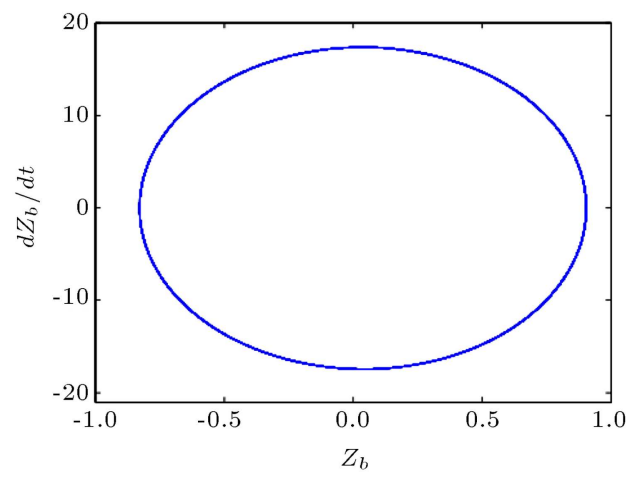

(a)

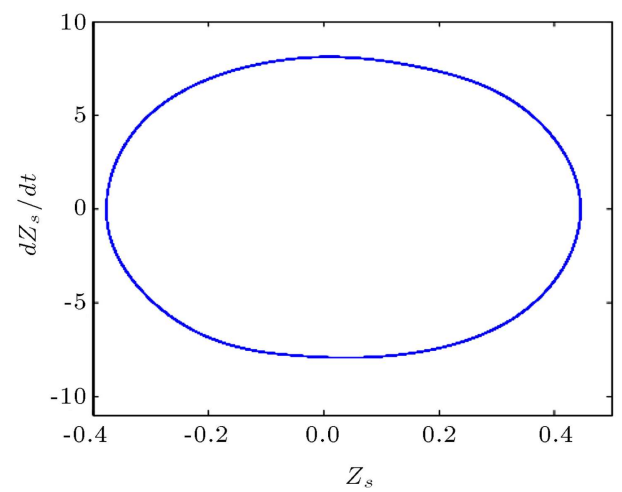

(c)

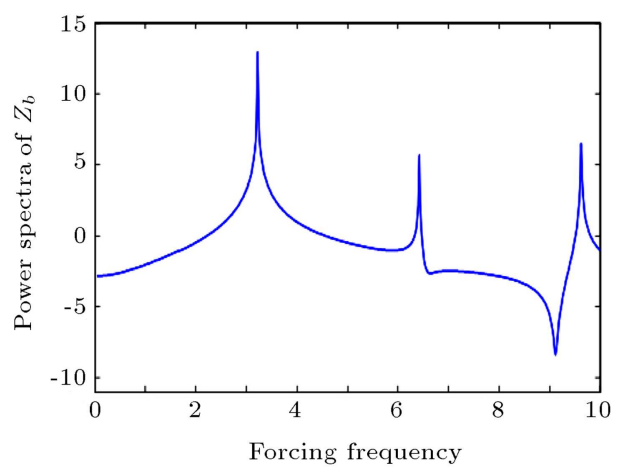

(b)

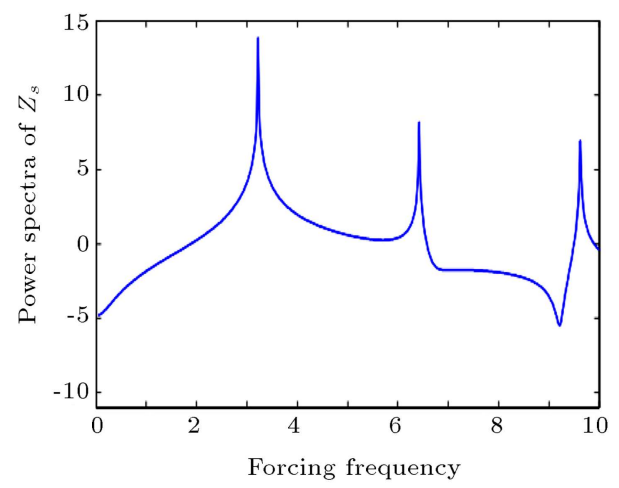

(d)

Figure 19. Phase plane portrait and power spectrum of $Z_{b}(t)$ and $Z_{s}(t)\left(k_{b}=25000 \mathrm{~N} / \mathrm{m}, k_{s f}=50000 \mathrm{~N} / \mathrm{m}, A=0.06 \mathrm{~m}\right.$, $\left.f=3.2 \mathrm{~Hz}, \alpha=35^{\circ}, \beta=9^{\circ}\right)$.

passengers were added to the seven-d.o.f model, considerable differences in the dynamic behavior between the two models were shown. Also, the physical control parameters, such as the spring stiffness of the driver's seat $\left(k_{b}\right)$, the damping coefficient of driver's seat $\left(c_{b}\right)$, and the spring stiffness of the suspension $\left(k_{s f}\right)$, were used to eliminate the chaotic vibration, whereas the previous control parameters $(\alpha, \beta)$ were fixed in the chaotic regions. The results showed that by changing the stiffness and damping coefficients of the suspension and seat and by selecting the appropriate values, one can easily eliminate the chaos or minimize the vibrations of the vehicle and enhance the operational life of the system. These results can give some insight into designers and engineers works when selecting appropriate basic vehicle suspension parameters based on nonlinear model considerations.

\section{Nomenclature}

$m_{s} \quad$ Sprung mass

$m_{a}, m_{b}, m_{c}, m_{d}$ Passenger and seat mass

$I_{\varphi}$

Roll axis moment of inertia

$I_{\theta}$

Pitch axis moment of inertia

$m_{u f}$

$m_{u r}$
$Z_{s}(t)$

$\varphi(t)$

$\theta(t)$

$Z_{a}(t)$

$Z_{b}(t)$

$Z_{c}(t)$

$Z_{d}(t)$

$Z_{u f l}(t)$

$Z_{u f r}(t)$

$Z_{u r l}(t)$

$Z_{u r r}(t)$

$k_{s f}$

$k_{s r}$

$k_{a}, k_{b}, k_{c}, k_{d}$

$n_{s f}, n_{s r}$

$c_{\text {suf }}$
Displacement of sprung mass

Roll angular displacement of sprung mass

Pitch angular displacement of sprung mass

Displacement of seat $(a)$

Displacement of seat $(b)$

Displacement of seat $(c)$

Displacement of seat $(d)$

Displacement of front-left unsprung mass

Displacement of front-right unsprung mass

Displacement of rear-left unsprung mass

Displacement of front-right unsprung mass

Front suspension spring stiffness

Rear suspension spring stiffness

Seat spring stiffness

Nonlinear coefficient of suspension spring

Damping coefficient of front suspension for tension 


\begin{tabular}{|c|c|}
\hline$c_{s d f}$ & $\begin{array}{l}\text { Damping coefficient of front suspension } \\
\text { for compression }\end{array}$ \\
\hline$c_{\text {sur }}$ & $\begin{array}{l}\text { Damping coefficient of rear suspension } \\
\text { for tension }\end{array}$ \\
\hline$c_{s d r}$ & $\begin{array}{l}\text { Damping coefficient of rear suspension } \\
\text { for compression }\end{array}$ \\
\hline$c_{a}, c_{b}, c_{c}, c_{d}$ & Damping coefficient of seat \\
\hline$k_{u s f}, k_{u s r}$ & Tire spring stiffness \\
\hline$c_{u s f}, c_{u s r}$ & Damping coefficient of tire \\
\hline$n_{u s f}, n_{u s r}$ & Nonlinear coefficient of tire spring \\
\hline$a$ & $\begin{array}{l}\text { Length between the front of vehicle } \\
\text { and the center of gravity of sprung } \\
\text { mass }\end{array}$ \\
\hline$b$ & $\begin{array}{l}\text { Length between the rear of vehicle and } \\
\text { the center of gravity of sprung mass }\end{array}$ \\
\hline$s$ & Width of sprung mass \\
\hline$e$ & $\begin{array}{l}\text { Latitude distance between front seats } \\
\text { and the center of gravity of sprung } \\
\text { mass }\end{array}$ \\
\hline$f$ & $\begin{array}{l}\text { Longitude distance between seats and } \\
\text { the center of gravity of sprung mass }\end{array}$ \\
\hline$h$ & $\begin{array}{l}\text { Longitude distance between rear seats } \\
\text { and the center of gravity of sprung } \\
\text { mass }\end{array}$ \\
\hline
\end{tabular}

\section{References}

1. Robson, J.D. "Road surface description and vehicle response", International Journal of Vehicle Design, $\mathbf{9}$, pp. 25-35 (1979).

2. Williams, R.A. "Automotive active suspensions, Part 1: basic principles", Proceedings of the Institute of Mechanical Engineers, Journal of Automobile Engineering, Proceedings Part D, 211, USA, pp. 415-426 (1997).

3. Verros, G., Natsiavias, S. and Stepan, G. "Control and dynamics of quarter-car models with dual-rate damping", Journal of Vibration and Control, 6, pp. 1045-1063 (2000).

4. Yang, J., Suematsu, Y. and Kang, Z. "Two-degree-offreedom controller to reduce the vibration of vehicle engine-body system", IEEE Transactions on Control Systems Technology, 9, pp. 295-317 (2001).

5. Moran, A. and Nagai, M. "Optimal active control of nonlinear vehicle suspension using neural networks", JSME International Journal, Series C, 37, pp. 707718 (1994).

6. Vetturi, D., Gadola, M., Cambiaghi, D. and Manzo, L., "Semi-active strategies for racing car suspension control", II Motorsports Engineering Conference and Exposition, Dearborn, USA (1996).

7. Campos, J., Davis, L., Lewis, F.L., Ikenaga, S., Scully, S. and Evans, M. "Active suspension control of ground vehicle heave and pitch motions", Proceedings of the
Seventh IEEE Mediterranean Control Conference on Control and Automation, Haifa, Israel (1999).

8. Ikenaga, S., Lewis, F.L., Campos, J. and Davis, L. "Active suspension control of ground vehicle based on a full-vehicle model", Proceedings of America Control Conference, Chicago, IL, USA (2000).

9. Choi, S.B. and Lee, S.K. "A hysteresis model for the field dependent damping force of a magnetorheological damper", Journal of Sound and Vibration, 24, pp. 361-375 (2001).

10. Lai, C.Y. and Liao, W.H. "Vibration control of a suspension system via magneto-rheological fluid damper", Journal of Vibration and Control, 8, pp. 515-527 (2002).

11. Liu, H., Nonami, K. and Hagiwara, T. "Semi-active fuzzy sliding mode control of full vehicle and suspensions", Journal of Vibration and Control, 11, pp. 10251042 (2005).

12. Litak, G., Borowiec, M., Ali, M., Saha, L.M. and Firswell, M.I. "Pulsive feedback control of a quarter car model forced by a road profile", Journal of Chaos, Solition and Fractals, 33, pp. 1672-1676 (2007).

13. Litak, G., Borowiec, M., Firswell, M.I. and Szabelski, K. "Chaotic vibration of a quarter-car model excited by the road surface profile", Communications in Nonlinear Science and Numerical Simulation, 13, pp. 13731383 (2008).

14. Litak, G., Borowiec, M., Firswell, M.I. and Przystupa, W. "Chaotic response of a quarter car model forced by a road profile with stochastic component", Journal of Chaos, Solitions and Fractals, 39, pp. 2448-2456 (2009).

15. Samandari, H. and Rezaee, M. "The stability and chaos analysis of a nonlinear wheeled vehicle model under road excitation", Proceedings of the ASME 2010 10th Biennial Conference on Engineering Systems Design and Analysis ESDA2010, Istanbul, Turkey (2010).

16. Singru, R.M. and Naik, P.D. "Resonance, stability and chaotic vibration of a quarter-car vehicle model with time-delay feedback", Commun Nonlinear Sci. Numer. Simulat., 16, pp. 3397-3410 (2011).

17. Zhu, Q. and Ishitobi, M. "Chaos and bifurcations in a nonlinear vehicle model", Journal of Sound and Vibration, 275, pp. 1136-1146 (2004).

18. Zhu, Q. and Ishitobi, M. "Chaotic vibration of a nonlinear full-vehicle model", International Journal of Solids and Structures, 43, pp. 747-759 (2006).

19. Fakhraei, J., Khanlo, H.M. and Ghayour, M. "Analysis of the passengers effect on chaotic vibrations of a nonlinear full vehicle model", Modares Mechanical Engineering (In Persian), 15, pp. 173-184 (2015).

20. Sedighi, M.H. and Shirazi, H.K. "Bifurcation analysis in hunting dynamical behavior in a railway bogie: Using novel exact equivalent functions for discontinuous nonlinearities", Scientia Iranica, 19, pp. 1493-1501 (2012). 
21. Belato, D., Weber, H.I., Balthazar, J.M. and Mook, M.D.T. "Chaotic vibration of a nonideal electromechanical system", International Journal of Solids and Structures, 38, pp. 1699-1706 (2001).

22. Zhu, Q., Tani, J. and Takagi, T. "Chaotic vibrations of a magnetically levitated system with two degrees of freedom", Journal of Technical Physics, 35, pp. 171184 (1994).

23. Pust, L. and Szöllös, O. "The forced chaotic and irregular oscillations of the nonlinear two degrees of freedom (2dof) system", International Journal of Bifurcation and Chaos, 9, pp. 479-491 (1999).

24. Nayfeh, A.H. and Balachandran, B., Applied Nonlinear Dynamics, John Wiley and Sons, Inc., New York, USA, pp. 525-537 (1995).

25. Xu, J. "Some advances on global analysis of nonlinear systems", Chaos, Solitons and Fractals, 39, pp. 18391848 (2009).

26. Wolf, A., Swift, J.B., Swinney, H.L. and Vastano, J.A. "Determining Lyapunov exponents from a time series", Physica D, 16, pp. 285-317 (1985).

\section{Biographies}

Javad Fakhraei was born in 1987 in Iran. After studying in Islamic Azad University of Khomeynishahr, he received a BS degree in Mechanical Engineeringsolid Mechanics in 2010. He pursued his study in Mechanical Engineering in Isfahan University of
Technology (IUT) and obtained his MS degree in 2015. He focused on the nonlinear dynamic behaviors and chaotic vibrations of vehicles with passive and active suspensions during his MS course. His general academic areas of interest include nonlinear dynamical systems, chaotic vibrations and vehicle dynamics.

Heshmat Mohammad Khanlo received BS degree in Aerospace Engineering from Shahid Sattari Aeronoutical University of Science and Technology in 1995 and MSc degree in Mechanical Engineering from Isfahan University of Technology in 2003. He received his $\mathrm{PhD}$ degree in Mechanical Engineering from Isfahan University of Technology, in 2011. His research interests are system dynamics, control systems, structural dynamics, nonlinear vibration, and chaos and aircraft systems.

Mostafa Ghayour received his MSc degree in Mechanical Engineering from Tehran University, Iran, in 1978. He received the $\mathrm{PhD}$ degree from Isfahan University of Technology, Isfahan, Iran in 1998. He is a Professor at Mechanical Engineering Department, Isfahan University of Technology, Isfahan, Iran, (IUT). His research interests are on robotics, control systems, and mechanical vibrations. He has published a few papers in international journals that can be seen in his personal web page. 